\title{
Multi-frequency radio continuum mapping of giant radio galaxies
}

\author{
K.-H. Mack ${ }^{1, \star}$, U. Klein ${ }^{1}$, C.P. O’Dea ${ }^{2}$, and A.G. Willis ${ }^{3}$ \\ 1 Radioastronomisches Institut, Universität Bonn, Auf dem Hügel 71, 53121 Bonn, Germany \\ 2 Space Telescope Science Institute, 3700 San Martin Drive, Baltimore, MD 21218, U.S.A. \\ 3 Dominion Radio Astrophysical Observatory, P. O. Box 248, Penticton, BC, Canada V2A 6K3, Canada
}

Received August 23; accepted October 8, 1996

\begin{abstract}
The giant radio galaxies NGC 315, DA 240, 3C 236, 3C 326, and NGC 6251 have been observed at $92 \mathrm{~cm}$ and $49 \mathrm{~cm}$ wavelengths with the Westerbork Synthesis Radio Telescope (WSRT) and at $\lambda \lambda 11 \mathrm{~cm}$, $6.3 \mathrm{~cm}$, and $2.8 \mathrm{~cm}$ using the Effelsberg 100-m telescope. These objects all exhibit strong polarized radio emission across the entire radio frequency domain. Their spectral index distributions are very complex, with significant variations across individual objects.
\end{abstract}

Key words: galaxies: individual: NGC 315; DA 240; 3C 326; 3C 236; NGC 6251; radio continuum: galaxies

\section{Introduction}

Giant radio galaxies (GRGs) form an extreme class of extragalactic radio sources. They can be studied in detail because of their large angular size. Their huge intrinsic sizes must be due either to very powerful AGNs or to a surrounding medium of very low density. These large sizes imply long evolution times which can be investigated by multi-frequency observations. This type of source also allows us to study characteristics of the intergalactic medium as the source expands into the host galaxy's surrounding environment.

Numerous studies of GRGs have been performed especially in the low-frequency regime (e.g. Willis \& Strom 1978; Willis et al. 1978; 1981; Bridle et al. 1979; Strom \& Willis 1980; Barthel et al. 1985; Jägers 1986; 1987a,b). At higher frequencies there have been measurements with the Effelsberg $100-\mathrm{m}$ telescope at $\lambda \lambda 11 \mathrm{~cm}$ and $6 \mathrm{~cm}$ by Baker et al. (1974), Stoffel \& Wielebinski (1978),

Send offprint requests to: K.-H. Mack, Bologna address

* Present address: Istituto di Radioastronomia del C.N.R., Via P. Gobetti 101, 40129 Bologna, Italy.
Strom et al. (1981), Klein et al. (1994), and Saripalli et al. (1996).

Since particle aging first affects higher electron energies, low-frequency observations mainly show the unevolved stage of the sources. Knowledge of the lowfrequency spectral indices is essential to fit spectral aging models, which will be reported in forthcoming papers.

High-frequency observations as reported by Klein et al. (1994) and Saripalli et al. (1996) are essentially free of Faraday effects and thus enable us to directly "map" the intrinsic (projected) magnetic field. A comparison of these observations with the low-frequency measurements allows us to determine the degree of disorder and (de-)polarization characteristics. The very high degree of polarization which is a general characteristic of the sources chosen in this study provide significant polarization information even at $326 \mathrm{MHz}$.

Here we report observations of the GRGs NGC 315, DA 240, 3C 236, 3C 326, and NGC 6251 at $\lambda \lambda 92 \mathrm{~cm}$ and $49 \mathrm{~cm}$, carried out with the Westerbork Synthesis Radio Telescope (WSRT) and $\lambda \lambda 11 \mathrm{~cm}, 6.3 \mathrm{~cm}$, and $2.8 \mathrm{~cm}$ using the Effelsberg 100-m telescope. The WSRT observations and results had previously been presented in brief by Willis \& O'Dea (1990). The 10.6-GHz data had been published by Klein et al. (1994), but the maps had not yet been cleaned with the antenna pattern, which has been performed in the meantime.

In Sect. 2 we describe the observations and the data analysis. In Sect. 3 the maps of total intensity and linear polarization are presented, along with a brief description of their most striking characteristics. In Sect. 4 we have compiled the integrated flux densities of the sources and source components to derive their integrated spectra. More detailed studies will be presented in forthcoming papers.

Throughout this paper we use $H_{0}=75 \mathrm{~km} \mathrm{~s}^{-1} \mathrm{Mpc}^{-1}$ and $q_{0}=1$. 
Table 1. Map parameters of the WSRT maps

\begin{tabular}{|c|c|c|c|c|c|c|c|c|c|c|}
\hline \multirow[t]{3}{*}{ Source } & \multicolumn{2}{|c|}{ Phase centre } & \multicolumn{4}{|c|}{ Beam size } & \multicolumn{4}{|c|}{ rms noise } \\
\hline & $\alpha_{50}$ & $\delta_{50}$ & $(326$ & $\mathrm{MHz})$ & $(60$ & $\mathrm{MHz}$ & & {$[\mathrm{mJ}$} & b.a. & \\
\hline & {$[\mathrm{h}: \mathrm{m}: \mathrm{s}]$} & {$\left[{ }^{\circ}:{ }^{\prime}:{ }^{\prime \prime}\right]$} & {$[\alpha]^{\prime \prime}$} & $\left.\times \delta\left[^{\prime \prime}\right]\right]$ & $\alpha{ }^{[\prime}$ & $\left.\times \delta\left[^{\prime \prime}\right]\right]$ & $\sigma_{\mathrm{I}}^{92}$ & $\sigma_{\mathrm{I}_{\mathrm{p}}}^{92}$ & $\sigma_{\mathrm{I}}^{49}$ & $\sigma_{\mathrm{I}_{\mathrm{p}}}^{49}$ \\
\hline NGC 315 & 005748.0 & 302059.9 & 55 & 110 & 28 & 55 & 1.7 & 0.8 & 0.6 & 0.5 \\
\hline DA 240 & $\begin{array}{lll}07 & 491.9\end{array}$ & 555028.9 & 55 & 67 & 28 & 34 & 2.0 & 1.2 & 0.6 & 0.5 \\
\hline $3 \mathrm{C} 236$ & 100656.1 & 344520.5 & 55 & 96 & 28 & 48 & 1.8 & 0.8 & 0.5 & 0.4 \\
\hline $3 \mathrm{C} 326$ & 155149.0 & 200504.2 & 55 & 161 & 28 & 81 & 2.7 & 3.1 & 1.0 & 0.6 \\
\hline NGC 6251 & 163436.8 & 822906.3 & 55 & 55 & 28 & 28 & 1.5 & 1.1 & 0.4 & 0.3 \\
\hline
\end{tabular}

Table 2. Map parameters of the Effelsberg maps

\begin{tabular}{|c|c|c|c|c|c|c|c|c|c|c|}
\hline \multirow[t]{2}{*}{ Source } & \multicolumn{2}{|c|}{ map centre $(2.7,4.8 \mathrm{GHz})$} & \multicolumn{2}{|c|}{ map centre $(10.6 \mathrm{GHz})$} & \multicolumn{6}{|c|}{ rms noise } \\
\hline & {$\left[\begin{array}{c}\alpha_{50} \\
{\left[{ }^{\mathrm{h}}:{ }^{\mathrm{m}}:{ }^{\mathrm{s}}\right]}\end{array}\right.$} & {$\left[{ }^{\circ}:^{\delta_{50}}:{ }^{\prime \prime}\right]$} & {$\left[\begin{array}{l}\alpha_{50} \\
{\left[{ }^{\mathrm{h}}:{ }^{\mathrm{m}}:{ }^{\mathrm{s}}\right]}\end{array}\right.$} & 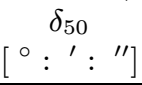 & $\sigma_{\mathrm{I}}^{11}$ & $\sigma_{\mathrm{I}_{\mathrm{p}}}^{11}$ & $\sigma_{\mathrm{I}}^{[\mathrm{m}}$ & $\begin{array}{l}\text { /b.a.] } \\
\sigma_{\mathrm{I}_{\mathrm{p}}}^{6.3}\end{array}$ & $\sigma_{\mathrm{I}}^{2.8}$ & $\sigma_{\mathrm{I}_{\mathrm{p}}}^{2.8}$ \\
\hline NGC 315 & 005546.4 & $\begin{array}{lll}30 & 01 & 42\end{array}$ & 005545.0 & 300000 & 4.9 & 2.1 & 2.4 & 1.2 & 1.4 & 0.4 \\
\hline DA 240 & 074419.2 & 555632 & 074434.0 & 555647 & 4.8 & 2.6 & 1.8 & 1.9 & 1.2 & 0.5 \\
\hline $3 \mathrm{C} 236$ & 100302.3 & 350857 & 100306.0 & 350846 & 4.1 & 1.7 & 2.2 & 1.2 & 1.2 & 0.4 \\
\hline $3 \mathrm{C} 326$ & 154937.3 & 201437 & 154941.5 & 201349 & 4.2 & 1.8 & 2.1 & 0.9 & 1.0 & 0.5 \\
\hline NGC 6251 & 164001.8 & 823016 & 164259.0 & 823257 & 3.4 & 2.2 & 3.4 & 1.3 & 1.1 & 0.4 \\
\hline
\end{tabular}

\section{Observations and data reduction}

\subsection{WSRT data}

All galaxies were observed by the WSRT in full redundancy mode; the correlator sampled both parallel and crossed dipole configurations. The parallel dipole data (WSRT channels XX and YY) were used to produce high dynamic range total intensity maps with the aid of the NRAO AIPS package as follows; the WSRT data were loaded into AIPS and first processed with the special AIPS task REDUN. REDUN is a translation into AIPS of the corresponding Dwingeloo DWARF program. It computes telescope dependent amplitude and phase errors by analyzing the observed amplitudes and phases of the many redundant baselines in the Westerbork array. After this initial operation has been done, WSRT observations can be further processed into high dynamic range images using the AIPS programs MX and ASCAL as has been described by Perley (1986). The signals in the crossed dipole channels XY and YX may be combined with the difference in signal between XX and YY channels to yield maps of the Stokes polarization parameters $Q$ and $U$. At $326 \mathrm{MHz}$ electric vector position angles can be rotated from their intrinsic position angles by many tens of degrees (more than 100 degrees is not uncommon) because of Faraday rotation within the ionosphere. Therefore all observations were first corrected for ionospheric Faraday rotation using the method developed at Dwingeloo by Spoelstra (1981). The flux calibration scale is that of Baars et al. (1977). In Table 1 we have compiled relevant observational parameters for the WSRT maps.

\subsection{Effelsberg data}

The high-frequency observations have been carried out with the Effelsberg $100-\mathrm{m}$ telescope using the $2.7-\mathrm{GHz}$ 1-horn, 3-channel receiver, the 4.8-GHz 2-horn, 3-channel, and the $10.6-\mathrm{GHz}$ 4-horn, 8-channel receiver system, all installed in the secondary focus of the telescope. The observations with the 10.6-GHz system have been described in detail by Klein et al. (1994). At $2.7 \mathrm{GHz}$ and $4.8 \mathrm{GHz}$ we had to use the single-beam mode (owing to limited observing time allocation), which is more strongly affected by bad weather conditions and terrestrial interference. The maps were large enough to cover the source plus some emission-free areas used for determination of zero levels and noise. Contrary to the multi-beam technique, which requires scanning in the horizontal system, the $2.7-\mathrm{GHz}$ and $4.8-\mathrm{GHz}$ maps have been obtained by scanning alternately along, and perpendicular to, the position angle of the source. The drive rates were $2^{\circ} / \mathrm{min}$ at $2.7 \mathrm{GHz}$ and $1^{\circ} / \mathrm{min}$ at $4.8 \mathrm{GHz}$, the scan interval $2^{\prime}$ and $1^{\prime}$, respectively. The individual maps were edited to diminish the influence of weather or terrestrial interference before they were averaged to yield final maps of Stokes $I, Q$, and $U$, employing the Fourier filter technique of Emerson \& Gräve (1988). The 10.6-GHz maps shown by Klein et al. (1994) have been CLEANed, applying the algorithm described by Klein \& Mack (1995). The 4.8-GHz maps have also been CLEANed, but the algorithm is more complicated in the case of maps not observed in the horizontal system so that some residual artifacts introduced by the antenna pattern may be left. 
Table 2 summarizes the relevant map parameters for each source. The data have been calibrated applying the scale of Baars et al. (1978). Because of the relatively large number of maps, where parts of them had to be blanked because of weather or interference effects, the noise level may vary significantly across the final maps. This is of special importance for the calculation of the polarized intensity maps. These have been produced as suggested by Wardle \& Kronberg (1974). Since the polarization information represents a pseudovector where neither the amplitude nor the phase has a Gaussian probability distribution one has to apply a correction term, especially in the case of polarized low-brightness regions that we are concerned with. The best estimate of the true polarized intensity can be calculated as

$I_{\mathrm{P}}=\sqrt{Q^{2}+U^{2}-\left(1.2 \sigma_{Q U}\right)^{2}}$

where $Q, U$ is the intensity in the Stokes $Q$ - and $U$-map, respectively, and $\sigma_{Q U}$ is the mean value of the noise in the $Q$ - and $U$-maps. The factor 1.2 has been found empirically to be best suited to shift the peak of the (positive) noise distribution function to zero.

In view of this correction it is clear that the determination of the proper noise value is very important to obtain the true polarized intensity. Therefore we have developed a routine which calculates the noise at each map pixel as a function of the number of individual maps (i.e. integration time) to be averaged at this pixel. The polarized intensity is thus calculated by accounting for the inhomogeneous distribution of the noise level.

\section{The maps}

In the following we present the radio continuum maps of the GRGs. The results for each source are discussed individually. We show two maps per source and frequency, one displaying the total intensity, with vectors proportional to the polarized intensity, the other giving the polarized intensity as contours, with vectors proportional to the percentage polarization. The angle of the vectors is the direction of the electric field. For magnetic field maps we refer to Klein et al. (1994). Detailed studies of spectral indices, rotation, and depolarization measures will be presented in forthcoming papers. We have also compiled a number of point sources observed in the galaxies' fields which probably do not have any physical connection to the GRGs. Their coordinates and flux densities at $609 \mathrm{MHz}$ can be found in Table 3-6.

\section{1. $N G C 315$}

The first detailed study of this source was performed by Bridle et al. (1976) using the Arecibo telescope at $0.43 \mathrm{GHz}, 1.41 \mathrm{GHz}$, and $2.38 \mathrm{GHz}$. Stoffel \& Wielebinski (1978) made first measurements with the Effelsberg telescope at $2.7 \mathrm{GHz}$. Concentrating on the jet,
Bridle et al. (1979) observed NGC 315 at $609 \mathrm{MHz}$, $1.4 \mathrm{GHz}$, and $4.9 \mathrm{GHz}$ with the WSRT and the VLA. The most extensive study including the polarization data was performed by Willis et al. (1981). A new map at $609 \mathrm{MHz}$ was obtained by Jägers (1987a) using the upgraded WSRT.

Table 3. Point sources found in the NGC 315 field (at $609 \mathrm{MHz}$

\begin{tabular}{|c|c|c|c|c|}
\hline \multirow[t]{2}{*}{ No. } & \multicolumn{2}{|c|}{ coordinates } & \multirow{2}{*}{$\begin{array}{c}S_{609} \\
{[\mathrm{mJy}]}\end{array}$} & \multirow[t]{2}{*}{ comments } \\
\hline & {$\left[\begin{array}{l}\alpha_{50} \\
{\left[{ }^{\mathrm{h}}:{ }^{\mathrm{m}}:{ }^{\mathrm{s}}\right]}\end{array}\right.$} & {$\left[{ }^{\circ}:{ }^{\delta_{50}}:{ }^{\prime \prime}\right]$} & & \\
\hline 1 & 005345.3 & 295208.0 & $50.4 \pm 2.8$ & extended \\
\hline 2 & 005349.0 & 301951.8 & $7.0 \pm 2.1$ & \\
\hline 3 & 005352.6 & 300152.4 & $6.8 \pm 2.3$ & \\
\hline 4 & 005408.9 & 294256.9 & $32.8 \pm 2.2$ & \\
\hline 5 & 005419.7 & 301309.4 & $20.7 \pm 4.3$ & confused \\
\hline 6 & 005425.5 & $\begin{array}{lll}30 & 02 & 23.2\end{array}$ & $5.7 \pm 2.3$ & \\
\hline 7 & 005425.8 & 293851.3 & $9.5 \pm 2.0$ & \\
\hline 8 & 005441.9 & $\begin{array}{lll}30 & 16 & 31.7\end{array}$ & $27.6 \pm 2.4$ & \\
\hline 9 & 005520.3 & 293849.7 & $20.8 \pm 2.2$ & \\
\hline 10 & 005534.4 & 295449.6 & $5.0 \pm 2.2$ & \\
\hline 11 & 005544.5 & 295853.6 & $32.3 \pm 2.3$ & \\
\hline 12 & 005547.8 & 294034.6 & $29.5 \pm 2.4$ & extended \\
\hline 13 & 005550.4 & 294024.1 & $7.1 \pm 2.1$ & extended \\
\hline 14 & 005551.7 & 300642.5 & $4.0 \pm 2.2$ & \\
\hline 15 & 005554.0 & 302049.0 & $62.4 \pm 2.3$ & extended \\
\hline 16 & 005551.7 & 302012.8 & $38.2 \pm 2.3$ & extended \\
\hline 17 & 005557.7 & 294655.2 & $6.4 \pm 2.4$ & extended \\
\hline 18 & 005615.9 & 300657.8 & $11.9 \pm 2.7$ & extended \\
\hline 19 & 005631.2 & 294904.5 & $19.6 \pm 2.4$ & extended \\
\hline 20 & 005631.3 & $\begin{array}{lll}30 & 00 & 16.2\end{array}$ & $9.0 \pm 2.2$ & \\
\hline 21 & 005637.6 & 295610.1 & $17.6 \pm 2.2$ & \\
\hline 22 & 005644.9 & 300822.6 & $5.9 \pm 2.1$ & \\
\hline 23 & 005659.3 & 293802.9 & $39.4 \pm 2.2$ & \\
\hline 24 & 005703.5 & 293948.3 & $22.1 \pm 2.1$ & \\
\hline 25 & 005714.7 & $\begin{array}{lll}30 & 1149.7\end{array}$ & $21.7 \pm 2.2$ & \\
\hline 26 & 005715.1 & $\begin{array}{lll}30 & 06 & 08.2\end{array}$ & $6.0 \pm 2.0$ & \\
\hline 27 & 005739.2 & $\begin{array}{ll}30 & 0350.7\end{array}$ & $159.1 \pm 2.6$ & extended \\
\hline 28 & 005745.6 & 301859.0 & $23.7 \pm 2.4$ & \\
\hline 29 & 005754.8 & $\begin{array}{ll}30 & 0255.1\end{array}$ & $7.0 \pm 2.1$ & \\
\hline 30 & 005800.7 & 300236.6 & $13.5 \pm 2.2$ & \\
\hline
\end{tabular}

"Extended" means sources with a deconvolved axis in $\alpha \geq$ $30^{\prime \prime}$ or in $\delta \geq 58^{\prime \prime}$ of the fitted ellipse.

The overall structure of NGC 315 suggests precessing beams, although the two lobes are anything but symmetric. The south-eastern lobe is broad, with its northern extension having a steep spectrum: it is not visible at $10.6 \mathrm{GHz}$. In contrast, the north-western lobe is narrow, with its "back-flowing" extension having a remarkably flat spectrum as suggested by the high-frequency maps, in which it is still easily visible. This peculiar behaviour was already pointed out by Klein et al. (1994), and suggests that a simple precession model is not able to 
explain the overall morphology and spectral characteristics. While the jet feeding the north-western lobe is bright and permanently visible over the whole frequency range, the counter-jet is only visible intermittently. As will be shown in Sect. 4.1, the spectrum of NGC 315 and its individual components is difficult to determine reliably. In any case, the strong asymmetry of the source, both, in its lobe structure as well as in its jet/counter-jet lengths, strongly suggests different densities/pressures of the intergalactic medium on either side of the host galaxy, rather than an intrinsic cause.

\section{2. $D A 240$}

Willis et al. (1974) presented the first high-resolution observations of this source at $609 \mathrm{MHz}$. Baker et al. (1974) showed a $\lambda 11 \mathrm{~cm}$ map, obtained with the Effelsberg telescope. Spectral index studies have been performed by Strom et al. (1981) who compared maps at $\lambda \lambda 49 \mathrm{~cm}$ and $6 \mathrm{~cm}$. Tsien (1982) obtained 0.15 and 1.4-GHz maps of the entire source and high-frequency observations of the famous hot spot. New $609-\mathrm{MHz}$ observations were obtained by Jägers (1987b) with the 3-km WSRT.

Table 4. Point sources found in the DA 240 field (at $609 \mathrm{MHz}$ )

\begin{tabular}{|c|c|c|c|c|}
\hline \multirow[t]{2}{*}{ No. } & \multicolumn{2}{|c|}{ coordinates } & $S_{609}$ & comments \\
\hline & {$\left[\begin{array}{c}\alpha_{50} \\
\left.{ }^{\mathrm{h}}: \stackrel{\mathrm{m}}{\mathrm{m}}: \mathrm{s}\right]\end{array}\right.$} & {$\left[{ }^{\circ}::^{\delta_{50}}:{ }^{\prime \prime}\right]$} & [mJy] & \\
\hline 1 & 074332.5 & 554859.8 & $38.6 \pm 0.4$ & confused \\
\hline 2 & 074401.4 & 560836.6 & $6.6 \pm 0.2$ & \\
\hline 3 & 074413.0 & 554400.6 & $11.6 \pm 0.2$ & \\
\hline 4 & 074412.9 & 560438.5 & $55.8 \pm 0.2$ & \\
\hline 5 & 074423.2 & $\begin{array}{lll}56 & 15 & 18.0\end{array}$ & $17.9 \pm 0.2$ & \\
\hline 6 & 074437.1 & 560804.0 & $4.5 \pm 0.2$ & \\
\hline 7 & 074522.8 & 560550.4 & & confused \\
\hline 8 & 074556.9 & $\begin{array}{ll}56 & 0259.9\end{array}$ & & confused \\
\hline 9 & 074634.5 & $\begin{array}{lll}55 & 4259.4\end{array}$ & $11.9 \pm 0.3$ & extended \\
\hline 10 & 074649.6 & 555254.3 & $22.5 \pm 0.2$ & \\
\hline
\end{tabular}

"Extended" means sources with a deconvolved axis in $\alpha \geq$ $30^{\prime \prime}$ or in $\delta \geq 36^{\prime \prime}$ of the fitted ellipse.

At low frequencies DA 240 is the archetype of a fat double radio galaxy with almost circularly extended lobes, the western lobe having an additional protrusion in the south-western direction. At higher frequencies the lobes shrink to a small straight channel marking the active zone of the source. The hot spot in the eastern lobe, 4C 56.16, is the most salient feature at high frequencies.

\section{3. $3 C 236$}

The first high-resolution study was presented by Willis et al. (1974) using WSRT observations at $609 \mathrm{MHz}$.
Strom \& Willis (1980) studied the spectral index distribution between $609 \mathrm{MHz}, 1.4 \mathrm{GHz}$, and $4.8 \mathrm{GHz}$. The large and small scale structure of 3C 236 was the subject of a paper by Barthel et al. (1985) who included both, 1.4-GHz WSRT and VLBI observations for their study. Jägers (1987b) obtained a $609-\mathrm{MHz}$ map with the $3-\mathrm{km}$ WSRT.

At $326 \mathrm{MHz}$ the bridge between the outer lobe areas and the central core is almost closed. The source is very narrow. While most sources of this species reveal low-brightness protrusions in a lateral direction at low frequencies, 3C 236 maintains its straight direction without showing any large-scale curves, wiggles or bends. The observed polarization of the core is an instrumental artifact. The north-western lobe looks more complex in polarized intensity than does the south-eastern one. This is even more evident if one compares the orientations of the electric field vectors.

Table 5. Point sources found in the 3C 236 field (at $609 \mathrm{MHz}$ )

\begin{tabular}{|c|c|c|c|c|}
\hline \multirow[t]{2}{*}{ No. } & \multicolumn{2}{|c|}{ coordinates } & \multirow{2}{*}{$\begin{array}{c}S_{609} \\
{[\mathrm{mJy}]}\end{array}$} & \multirow[t]{2}{*}{ comments } \\
\hline & {$\left[\begin{array}{c}\alpha_{50} \\
{\left[{ }^{\mathrm{h}}:{ }^{\mathrm{m}}:{ }^{\mathrm{s}}\right]}\end{array}\right.$} & {$\left[\begin{array}{c}\delta_{50} \\
{\left[{ }^{\circ}:{ }^{\prime}:\right.}\end{array}\right.$} & & \\
\hline 1 & 100143.6 & 350400.2 & $60.5 \pm 0.7$ & \\
\hline 2 & 100149.3 & 350429.0 & $32.1 \pm 0.7$ & \\
\hline 3 & 100221.1 & $\begin{array}{lll}35 & 16 & 48.7\end{array}$ & & confused \\
\hline 4 & 100243.5 & 350540.6 & $24.4 \pm 0.7$ & \\
\hline 5 & 100246.6 & 345035.7 & $114.4 \pm 0.7$ & \\
\hline 6 & 100249.7 & 345446.8 & $9.9 \pm 0.7$ & \\
\hline 7 & 100251.3 & 351651.8 & $164.1 \pm 0.7$ & \\
\hline 8 & 100302.7 & 350427.4 & $13.3 \pm 0.7$ & \\
\hline 9 & 100309.4 & 350350.1 & $27.7 \pm 0.7$ & \\
\hline 10 & 100316.5 & 345642.7 & $422.1 \pm 0.7$ & \\
\hline 11 & 100336.6 & $\begin{array}{lll}35 & 11 & 24.9\end{array}$ & $9.1 \pm 1.0$ & \\
\hline 12 & 100400.4 & $\begin{array}{lll}35 & 09 & 22.7\end{array}$ & $19.3 \pm 1.0$ & \\
\hline 13 & 100407.2 & 345232.0 & $5.4 \pm 0.7$ & \\
\hline 14 & 100417.5 & 350934.9 & $8.4 \pm 0.8$ & \\
\hline 15 & 100417.7 & 345233.3 & $8.9 \pm 0.8$ & \\
\hline 16 & 100421.0 & 345935.9 & & confused \\
\hline 17 & 100428.7 & 345912.3 & & confused \\
\hline 18 & 100446.2 & 345329.7 & $154.6 \pm 0.9$ & \\
\hline 19 & 100450.6 & $\begin{array}{llll}35 & 17 & 47.8\end{array}$ & $10.0 \pm 0.8$ & \\
\hline 20 & 100454.1 & 351643.6 & $23.5 \pm 0.8$ & \\
\hline 21 & 100453.8 & 345636.8 & $17.7 \pm 0.7$ & \\
\hline
\end{tabular}

"Extended" means sources with a deconvolved axis in $\alpha \geq$ $30^{\prime \prime}$ or in $\delta \geq 51^{\prime \prime}$ of the fitted ellipse.

The overall morphology is characterized by the narrow extent of the lobes perpendicular to the source axis. Comparing the two lobes, however, the north-western one appears broader, a fact which becomes especially evident in the polarized intensity map. The bridge emission between the core and the outer lobes becomes fainter with increasing frequency, which results in a steepening of the 
spectrum and is indicative of aged particles, a typical behaviour in FRII-radio galaxies.

\section{4. $3 C 326$}

The first maps of 3C 326 were presented by Mackay (1969) at 408 and $1407 \mathrm{MHz}$. Bridle et al. (1972) and Baker (1974) published single-dish observations at $1.4 \mathrm{GHz}$. The most detailed analysis of this source has been performed by Willis \& Strom (1978) who presented maps at frequencies of $609 \mathrm{MHz}, 1.4$ and $5 \mathrm{GHz}$.

Table 6. Point sources found in the NGC 6251 field (at $609 \mathrm{MHz})$

\begin{tabular}{|c|c|c|c|c|}
\hline \multirow[t]{2}{*}{ No. } & \multicolumn{2}{|c|}{ coordinates } & \multirow{2}{*}{$\begin{array}{c}S_{609} \\
{[\mathrm{mJy}]}\end{array}$} & \multirow[t]{2}{*}{ comments } \\
\hline & {$\left[\begin{array}{c}\alpha_{50} \\
{\left[{ }^{\mathrm{h}}:{ }^{\mathrm{m}}:{ }^{\mathrm{s}}\right]}\end{array}\right.$} & {$\left[\begin{array}{c}\delta_{50} \\
{\left[{ }^{\circ}:{ }^{\prime}:\right.}\end{array}\right.$} & & \\
\hline 1 & 162253.7 & $8248 \quad 07.0$ & $23.2 \pm 0.1$ & \\
\hline 2 & 162302.0 & 823400.9 & $11.5 \pm 0.1$ & \\
\hline 3 & 162505.2 & 825531.3 & $35.8 \pm 0.1$ & \\
\hline 4 & $\begin{array}{lll}16 & 26 & 07.1\end{array}$ & 825004.6 & $7.7 \pm 0.1$ & \\
\hline 5 & 162643.0 & 823754.2 & $6.8 \pm 0.1$ & \\
\hline 6 & 162702.3 & $8238 \quad 12.8$ & $4.8 \pm 0.1$ & \\
\hline 7 & 162853.7 & 824345.7 & $7.9 \pm 0.1$ & \\
\hline 8 & 163008.4 & $\begin{array}{lll}82 & 46 & 16.1\end{array}$ & $6.5 \pm 0.2$ & \\
\hline 9 & 163049.6 & 824127.3 & $86.1 \pm 0.1$ & \\
\hline 10 & 163429.1 & 823814.4 & $9.6 \pm 0.1$ & \\
\hline 11 & 163528.7 & $\begin{array}{lll}82 & 46 & 19.9\end{array}$ & $54.5 \pm 0.3$ & extended \\
\hline 12 & 163809.4 & 824042.0 & $2.7 \pm 0.1$ & confused \\
\hline 13 & 164402.2 & 823521.6 & $5.2 \pm 0.1$ & \\
\hline 14 & 164413.2 & 822304.1 & $87.7 \pm 0.1$ & \\
\hline 15 & $1646 \quad 42.4$ & $\begin{array}{lll}82 & 39 & 21.7\end{array}$ & $101.7 \pm 0.1$ & \\
\hline 16 & 164704.9 & 820932.0 & $3.7 \pm 0.1$ & \\
\hline 17 & 164733.7 & 820824.4 & $4.2 \pm 0.1$ & \\
\hline 18 & 164815.5 & 820744.6 & $4.3 \pm 0.1$ & \\
\hline 19 & 164845.7 & 820909.8 & $5.3 \pm 0.1$ & \\
\hline 20 & 164859.8 & 820740.6 & $15.0 \pm 0.1$ & \\
\hline 21 & 165015.4 & $8217 \quad 15.7$ & $32.5 \pm 0.3$ & extended \\
\hline 22 & 165043.5 & 820939.4 & $152.0 \pm 0.1$ & \\
\hline 23 & 165137.8 & 822505.6 & $25.0 \pm 0.1$ & \\
\hline 24 & 165204.7 & 821507.0 & $25.0 \pm 0.2$ & confused \\
\hline 25 & 165313.0 & 822127.1 & $27.3 \pm 0.2$ & confused \\
\hline
\end{tabular}

"Extended" means sources with a deconvolved axis in $\alpha, \delta \geq$ $30^{\prime \prime}$ of the fitted ellipse.

The global structure of this source does not vary significantly at different frequencies. The western lobe is narrow, as is particularly evident at $10.6 \mathrm{GHz}$. The lowfrequency WSRT maps do not have sufficient north-south resolution to reveal this, owing to the relatively low declination of $3 \mathrm{C} 326$. The eastern lobe is extended perpendicular to the source axis with a narrow protrusion emerging to the east. The linear polarization maps disclose a more complex structure, with up to three components in the eastern lobe. The point sources at $\alpha_{50}=15^{\mathrm{h}} 48^{\mathrm{m}} 54^{\mathrm{s}} .7$, $\delta_{50}=20^{\circ} 11^{\prime} 01^{\prime \prime}$ and $\alpha_{50}=15^{\mathrm{h}} 49^{\mathrm{m}} 41^{\mathrm{s}} 0, \delta_{50}=20^{\circ} 18^{\prime} 18^{\prime \prime}$ are background sources as already noted by other authors. The eastern component of the western lobe is seen to broaden southwards. The western lobe also shows significant polarization $(p \sim 10 \%)$ in its central and western components, whereas the broader eastern component of this lobe is more weakly polarized. The large-scale isotropic orientation of the magnetic field is obvious over an extent of $6 ! 3$, corresponding to a linear size of $600 \mathrm{kpc}$.

The overall (projected) source morphology suggests an intrinsically rather symmetric structure, but with the eastern lobe probably bending away from its original orientation, perpendicular to the sky plane.

\section{5. $N G C 6251$}

This source was discovered by Waggett et al. (1977) who presented maps of the entire source or only the core at various frequencies between $38 \mathrm{MHz}$ and $15.4 \mathrm{GHz}$. Stoffel \& Wielebinski (1978) published a 2.7-GHz map observed with the Effelsberg telescope. The polarization characteristics have been studied by Willis et al. (1978). Detailed investigations of the jet have been performed by Saunders et al. (1981) and Perley et al. (1984) using high-frequency interferometric maps. Willis et al. (1982) presented a $609-\mathrm{MHz}$ map observed with the WSRT which has been superseeded by new $609-\mathrm{MHz}$ observations with the upgraded instrument (Jägers 1987a).

The north-western lobe appears to consist of two regions, a brighter one which extends symmetrically around the jet, and a fainter part which expands in a southwestern direction. This confirms the impression suggested by the $151-\mathrm{MHz}$ map of the north-western lobe (Waggett et al. 1977) that it is very extended in the south-western direction.

The faint part has a steep spectrum, indicating a higher particle age although parts of the very extended structure may have been filtered out in the interferometer maps, especially at $609 \mathrm{MHz}$. The strong gradients at the northern edges of both lobes delineate the genuine boundaries of the lobes. The jet can be traced out to about $10^{\prime}$. It consists of a luminous part up to $5^{\prime}$ from the core, and a fainter section which curves towards the hot spot. The counter-jet is detected close to the core along the first $3^{\prime}$, disappears and lights up again some $20^{\prime}$ further out. The $326-\mathrm{MHz}$ data confirm this feature being part of the counter-jet on its way to the hot spot as already suggested by Willis et al. (1982). Although less obvious in the $609-\mathrm{MHz}$ map, the connection of feature B to the south-eastern hot spot also becomes clear when this map is smoothed to the resolution of the $326-\mathrm{MHz}$ map. At the lower resolution our $609-\mathrm{MHz}$ map is also consistent with that obtained by Willis et al. (1978).

The high-frequency maps emphasize the most active parts of the radio galaxy, viz. the core, the jet, and the hot spots. Unfortunately, the $2.7-\mathrm{GHz}$ and $4.8-\mathrm{GHz}$ maps 
Table 7. Integrated flux densities of NGC 315

\begin{tabular}{|c|c|c|c|c|c|c|}
\hline No. & component & \multicolumn{5}{|c|}{$S_{\text {tot }}[\mathrm{mJy}]$} \\
\hline $1-10$ & total & $9706.6 \pm 123.6$ & $5332.1 \pm 95.2$ & $3373.3 \pm 89.8$ & $2461.2 \pm 74.9$ & $2194.4 \pm 81.1$ \\
\hline 1 & core+innerjet & $3521.3 \pm 42.3$ & $2432.4 \pm 33.6$ & $1602.4 \pm 26.7$ & $1434.3 \pm 22.8$ & $1303.2 \pm 25.7$ \\
\hline 2 & jet knot & $234.0 \pm$ & $119.2 \pm 9.3$ & $95.1 \pm 7.0$ & $47.1 \pm 5.7$ & $29.0 \pm 7.8$ \\
\hline 3 & hot spot & $1008.3 \pm 20.6$ & $595.7 \pm 18.8$ & $284.4 \pm 13.1$ & $229.7 \pm 11.4$ & $174.7 \pm 16.6$ \\
\hline 4 & bright lobe & $703.0 \pm 18.1$ & $501.2 \pm 17.8$ & $89.6 \pm 10.5$ & $147.3 \pm 10.9$ & $120.1 \pm 15.0$ \\
\hline 5 & weak lobe & $297.7 \pm 14.5$ & $109.1 \pm 14.5$ & $261.9 \pm 20.4$ & $42.2 \pm 9.6$ & $118.4 \pm 13.6$ \\
\hline 6 & back lobe & $1311.1 \pm 33.2$ & $529.9 \pm 31.7$ & $273.2 \pm 20.6$ & $149.2 \pm 18.6$ & $203.2 \pm 29.1$ \\
\hline 7 & counter-jet & $158.1 \pm 12.4$ & $62.7 \pm 12.5$ & $46.9 \pm 9.0$ & $13.6 \pm 8.1$ & $47.9 \pm 10.7$ \\
\hline 8 & bgs (counter-jet) & $152.8 \pm 16.4$ & $74.3 \pm 16.5$ & $44.8 \pm 13.7$ & & $8.5 \pm 15.0$ \\
\hline 9 & bright south eastern lobe & $1362.8 \pm 22.8$ & $613.8 \pm 19.5$ & $406.6 \pm 14.0$ & $261.9 \pm 11.8$ & $186.9 \pm 15.9$ \\
\hline 10 & weak south eastern lobe & $1009.4 \pm 28.2$ & $312.3 \pm 26.4$ & $207.0 \pm 21.4$ & $19.7 \pm 19.1$ & $36.2 \pm 25.0$ \\
\hline 11 & bgs1 south eastern & $316.0 \pm \quad 8.7$ & $154.4 \pm 8.6$ & $54.6 \pm 6.6$ & $52.2 \pm 4.7$ & $30.0 \pm 6.7$ \\
\hline 12 & bgs north western jet & $57.4 \pm \quad 6.8$ & $8.4 \pm 6.9$ & $8.5 \pm 4.7$ & $12.0 \pm 4.5$ & $15.0 \pm 5.7$ \\
\hline 13 & bgs2 south eastern & $152.8 \pm \quad 9.4$ & $18.8 \pm 9.2$ & $23.1 \pm 6.3$ & $11.8 \pm 6.1$ & $34.7 \pm 8.5$ \\
\hline
\end{tabular}

Table 8. Integrated flux densities of DA 240

\begin{tabular}{|r|l|r|r|r|r|r|}
\hline No. & component & \multicolumn{4}{|c|}{$S_{\text {tot }}[\mathrm{mJy}]$} \\
& & \multicolumn{1}{|c|}{$326 \mathrm{MHz}$} & \multicolumn{1}{c|}{$4750 \mathrm{MHz}$} & \multicolumn{1}{c|}{$2695 \mathrm{MHz}$} & \multicolumn{1}{c|}{$10550 \mathrm{MHz}$} \\
\hline $1-4$ & total & $17054.0 \pm 199.4$ & $9234.2 \pm 130.2$ & $2712.7 \pm 59.5$ & $1767.4 \pm 37.8$ & $1040.9 \pm 46.5$ \\
1 & eastern lobe & $10299.0 \pm 120.4$ & $5688.7 \pm 78.1$ & $1809.6 \pm 31.2$ & $1186.6 \pm 23.4$ & $749.3 \pm 27.8$ \\
2 & western lobe & $6109.8 \pm 84.8$ & $3147.1 \pm 60.2$ & $721.9 \pm 30.8$ & $456.5 \pm 19.4$ & $195.7 \pm 24.6$ \\
3 & core & $549.4 \pm 12.8$ & $374.3 \pm 11.2$ & $189.2 \pm 6.5$ & $121.9 \pm 3.9$ & $76.6 \pm 5.2$ \\
4 & bgs north western & $194.9 \pm 13.0$ & $67.4 \pm 11.3$ & & & $21.8 \pm 5.5$ \\
\hline
\end{tabular}

Table 9. Integrated flux densities of 3C 236

\begin{tabular}{|r|l|r|r|r|r|r|}
\hline No. & component & \multicolumn{4}{|c|}{$S_{\text {tot }}[\mathrm{mJy}]$} \\
& & \multicolumn{1}{|c|}{$326 \mathrm{MHz}$} & \multicolumn{1}{c|}{$4750 \mathrm{MHz}$} & \multicolumn{1}{c|}{$10550 \mathrm{MHz}$} \\
\hline $1-6$ & total & $13132.0 \pm 140.0$ & $8227.7 \pm 90.8$ & $3652.0 \pm 71.2$ & $2353.5 \pm 41.7$ & $1274.7 \pm 31.7$ \\
1 & core & $7409.5 \pm 76.0$ & $4999.4 \pm 51.9$ & $2355.5 \pm 31.1$ & $1579.1 \pm 19.3$ & $875.6 \pm 14.1$ \\
2 & western lobe & $588.1 \pm 22.3$ & $348.6 \pm 18.3$ & $153.0 \pm 18.3$ & $36.4 \pm 14.4$ & $40.3 \pm 13.6$ \\
3 & western hot spot & $1275.1 \pm 16.5$ & $761.1 \pm 11.5$ & $263.4 \pm 8.5$ & $176.4 \pm 6.7$ & $115.8 \pm 7.0$ \\
4 & eastern lobe & $1908.1 \pm 25.5$ & $1008.5 \pm 17.3$ & $353.3 \pm 13.3$ & $179.9 \pm 10.8$ & $75.3 \pm 10.7$ \\
5 & bgs east & $660.0 \pm 10.1$ & $444.1 \pm 7.8$ & $149.3 \pm 5.0$ & $152.2 \pm 5.0$ & $99.3 \pm 5.2$ \\
6 & eastern hot spot & $1062.6 \pm 18.6$ & $513.2 \pm 11.9$ & $283.2 \pm 13.4$ & $221.2 \pm 9.6$ & $76.7 \pm 8.9$ \\
7 & bgs south eastern & $342.1 \pm 12.7$ & $203.1 \pm 11.6$ & $106.1 \pm 9.4$ & $25.3 \pm 6.1$ & $10.6 \pm 6.7$ \\
8 & bgs south & $702.1 \pm 15.6$ & $408.0 \pm 11.6$ & & & $41.7 \pm 8.2$ \\
9 & bgs north & $306.4 \pm 10.6$ & $189.3 \pm 8.5$ & $76.0 \pm 6.0$ & $56.9 \pm 5.9$ & $29.2 \pm 6.5$ \\
\hline
\end{tabular}

Table 10. Integrated flux densities of 3C 326

\begin{tabular}{|c|c|c|c|c|c|c|}
\hline \multirow[t]{2}{*}{ No. } & \multirow[t]{2}{*}{ component } & \multicolumn{5}{|c|}{$\overline{S_{\text {tot }}[\mathrm{mJy}]}$} \\
\hline & & $326 \mathrm{MHz}$ & $609 \mathrm{MHz}$ & $2695 \mathrm{MHz}$ & $4750 \mathrm{MHz}$ & $10550 \mathrm{MHz}$ \\
\hline $1-5$ & total & $11955.4 \pm 128.0$ & $7398.2 \pm 108.9$ & $2222.1 \pm 73.8$ & $1301.2 \pm 13.3$ & $434.3 \pm 16.0$ \\
\hline 1 & eastern lobe & $6797.6 \pm 72.7$ & $4088.6 \pm 47.2$ & $1275.3 \pm 30.9$ & $717.9 \pm 7.3$ & $235.9 \pm 8.5$ \\
\hline 2 & centre & $3382.0 \pm 38.7$ & $2164.3 \pm 27.9$ & & $319.8 \pm 3.3$ & $86.7 \pm 6.4$ \\
\hline 3 & western lobe & $1534.6 \pm 22.9$ & $982.5 \pm 18.8$ & $933.6 \pm 43.7$ & $232.1 \pm 2.4$ & $114.1 \pm 5.6$ \\
\hline 4 & bgs west & $122.0 \pm 13.4$ & $62.6 \pm 12.1$ & & $15.6 \pm 0.5$ & \\
\hline 5 & bgs north & $129.1 \pm 10.0$ & $111.4 \pm 10.3$ & & $19.6 \pm 0.4$ & \\
\hline
\end{tabular}


had to be cut at their north-western and south-western edge (owing to limited observing time) but they can still yield valuable information on intensities and polarization characteristics of the major part of the source. For a complete 2.7-GHz map we refer to Stoffel \& Wielebinski (1978), which confirms our results. The polarization maps show that the north-western lobe is strongly polarized, with degrees of polarization as high as $70 \%(609 \mathrm{MHz})$. Polarized radiation is detected in most parts of the lobe. In contrast, the south-eastern lobe shows much less extended polarization, but again with high degrees $(\sim 50 \%)$. The jet is weakly polarized $(p<5 \%)$ close to the core. This suddenly changes beyond 3.3 where the map of polarized intensity shows its maximum. The fractional polarization increases to $23 \%$. It is striking that it coincides with the edge of the diffuse lobe emission. The orientation of the $\boldsymbol{E}$-vectors indicates the presence of magnetic fields coherent over several arcminutes at $326 \mathrm{MHz}$. At $609 \mathrm{MHz}$ these scales are even larger, suggesting the patchy structure of a depolarizing sheath.

\section{The integrated spectra}

We have determined the total flux densities of the entire sources and - where possible - of individual components. All maps, except the 2.7-GHz data, have been smoothed to a common beam size of $150^{\prime \prime} \times 150^{\prime \prime}$ (determined by the resolution at $4.8 \mathrm{GHz}$ ). In the case of $3 \mathrm{C} 326$ the final beam size in declination is defined by the original resolution of the $326-\mathrm{MHz}$ map $\left(150^{\prime \prime} \times 161^{\prime \prime}\right)$. At $2.7 \mathrm{GHz}$ we took the original beam of $261^{\prime \prime} \times 261^{\prime \prime}$. Because of this lower resolution it was difficult in some cases to integrate across the same areas. The larger beam may also lead to higher flux densities because of confusion. Their effect will disappear in most cases when determining spectral indices pixel by pixel which will be reported in a forthcoming paper. The $609-\mathrm{MHz}$ data are affected by missing-spacing effects, which implies that the flux densities are lower limits.

As already mentioned, the 2.7- and 4.8-GHz maps had to be cut in some cases. When that happened we did not give flux densities. The error calculation includes errors introduced by zero-level uncertainty, errors coming from the map noise, and calibration errors. Since systematic errors such as missing flux due to missing spacings, variable baselines, or different noise levels across the map cannot be included, the error ranges might be underestimated in some cases. Therefore, we also show the flux densities in graphic form and comment on the spectra where necessary. For the sake of clarity we do not show any spectra of the background sources (bgs). All individual areas used to integrate the various component flux densities, their numbers given in Tables 7-11, are shown shaded in the finding charts (Fig. 5).

\section{1. $N G C 315$}

The spectrum $\left(S_{\nu} \sim \nu^{-\alpha}\right)$ of the entire source is obviously dominated by the core which reveals a significant flattening towards higher frequencies $\left(\alpha_{\text {low }}=0.59 \pm 0.01\right.$; $\left.\alpha_{\text {high }}=0.12 \pm 0.01\right)$. Here $\alpha_{\text {low }}$ is the spectral index between 326 and $609 \mathrm{MHz}, \alpha_{\text {high }}$ is determined between 4.8 and $10.6 \mathrm{GHz}$. We have determined the spectrum of the south-eastern lobe in both the southern brighter and the northern fainter part. The spectrum of the brighter part is straight, with $\alpha=0.55 \pm 0.05$. The flux densities of the weak part are much more scattered, but the steeper spectral index of $\alpha=0.82 \pm 0.23$ is obvious.

If the $2.7-\mathrm{GHz}$ point is excluded, the bright western lobe has an almost straight spectrum, with $\alpha=0.53 \pm 0.04$. The determination of a spectrum of the weak part of the western lobe is not possible since the flux densities are too strongly distorted. The spectrum of the western hot spot flattens towards high frequencies $\left(\alpha_{\text {low }}=0.85 \pm 0.01\right.$; $\left.\alpha_{\text {high }}=0.34 \pm 0.01\right)$. The counter-jet region has a typical jet spectrum $(\alpha=0.60 \pm 0.25)$ up to $4.8 \mathrm{GHz}$. The bright knot within the jet has a spectral index of $\alpha=0.60 \pm 0.01$. The possible background source in the counter-jet area possesses a spectrum of $\alpha=0.70 \pm 0.24$, with a slight trend for a steepening towards higher frequencies. The spectrum of the huge backlobe of NGC 315 is still not well determined. The appearance of a typical spectrum with $\alpha=0.72 \pm 0.11$ between $326 \mathrm{MHz}$ and $4.8 \mathrm{GHz}$ is in contrast to the excess at $10.6 \mathrm{GHz}$, which has already been noticed by Klein et al. (1994), and is still significant even after CLEANing.

\section{2. $D A 240$}

The total spectrum is obviously dominated by the influence of the very bright hot spot within the eastern lobe, which shows a flattening of the spectral index towards higher frequencies $\left(\alpha_{\text {low }}=0.95 \pm 0.01 ; \alpha_{\text {high }}=0.58 \pm\right.$ $0.01)$. The spectra of the western lobe and the core are almost straight, with $\alpha=0.98 \pm 0.01$ and $\alpha=0.56 \pm 0.01$, respectively. The slight relative excess of the $2.7-\mathrm{GHz}$ flux density can be explained by confusion of the core region when observed with the $261^{\prime \prime} \times 261^{\prime \prime}$ beam.

\section{3. $3 C 236$}

The spectrum of the entire source shows a deficit of the $609-\mathrm{MHz}$ value, which can be explained by missing spacings of the interferometer. The core spectrum shows excessive 2.7- and 4.8-GHz values, most likely because of confusion of the western lobe, and possibly owing to CLEAN artifacts. The overall core spectrum between $326 \mathrm{MHz}$ and $10.6 \mathrm{GHz}$ is $\alpha=0.61 \pm 0.01$.

The eastern lobe spectrum reveals similar problems. The $609-\mathrm{MHz}$ flux density is too low relative to the other values, which can again be explained by missing zero spacings. The $2.7-\mathrm{GHz}$ and maybe also the $4.8-\mathrm{GHz}$ 
Table 11. Integrated flux densities of NGC 6251

\begin{tabular}{|c|c|c|c|c|c|c|}
\hline \multirow[t]{2}{*}{ No. } & \multirow[t]{2}{*}{ component } & \multicolumn{5}{|c|}{$S_{\text {tot }}[\mathrm{mJy}]$} \\
\hline & & $326 \mathrm{MHz}$ & $609 \mathrm{MHz}$ & $2695 \mathrm{MHz}$ & $4750 \mathrm{MHz}$ & $10550 \mathrm{MHz}$ \\
\hline $1-8$ & total & $11551.0 \pm 175.9$ & $6930.4 \pm 123.4$ & & & $1548.4 \pm 55.0$ \\
\hline 1 & core + jet & $4739.9 \pm 52.1$ & $3390.8 \pm 37.8$ & $2098.0 \pm 27.2$ & $1637.6 \pm 18.7$ & $1129.5 \pm 14.3$ \\
\hline 2 & western lobe & $3120.9 \pm 85.8$ & $1595.5 \pm 63.4$ & & & $213.5 \pm 26.4$ \\
\hline 3 & hot spot western lobe & $572.0 \pm 10.9$ & $388.1 \pm \quad 7.8$ & $170.0 \pm 4.8$ & $116.2 \pm 3.9$ & $70.2 \pm 4.3$ \\
\hline 4 & bgs1 western lobe & $208.1 \pm \quad 6.7$ & $125.4 \pm \quad 5.1$ & & $21.8 \pm 2.6$ & $16.3 \pm 3.0$ \\
\hline 5 & bgs2 western lobe & $24.1 \pm \quad 6.4$ & $7.6 \pm \quad 4.2$ & & & \\
\hline 6 & counter-jet & $323.5 \pm 33.0$ & $121.7 \pm 25.7$ & $24.6 \pm 16.9$ & & $47.1 \pm 13.5$ \\
\hline 7 & hot spot eastern lobe & $205.0 \pm \quad 8.2$ & $143.2 \pm \quad 6.1$ & $107.6 \pm 6.3$ & $4.4 \pm 3.5$ & $2.2 \pm 3.5$ \\
\hline 8 & eastern lobe & $2402.8 \pm 64.4$ & $1365.3 \pm 48.4$ & & & $8.7 \pm 25.3$ \\
\hline
\end{tabular}

point suffer from confusion with the core emission. If the 4.8-GHz point is regarded as real, a slight convex curvature of $\Delta \alpha=0.2$ is indicated. Between $326 \mathrm{MHz}$ and $10.6 \mathrm{GHz}$ a spectral index of $\alpha=0.93 \pm 0.01$ has been derived. The western lobe spectrum $(\alpha=0.77 \pm 0.01)$ has also been determined using the measurements at the lowest and highest frequency only, since the $2.7-\mathrm{GHz}$ flux density is too high (confusion) and the $4.8-\mathrm{GHz}$ value is uncertain because of an artificial depression in this part of the map. The western hot spot again reveals a slight flattening $\left(\alpha_{\text {low }}=0.83 \pm 0.01 ; \alpha_{\text {high }}=0.53 \pm 0.01\right)$. The measurements in the eastern hot spot area and of the background source west of the hot spot are too uncertain to determine the overall spectrum, owing to confusion.

\section{4. $3 C 326$}

All spectra determined here show a clear steepening towards higher frequencies. The low- and high-frequency spectral indices have been compiled in Table 12 .

Table 12. Spectral indices of $3 \mathrm{C} 326$ (Errors are in all cases $\leq 0.01)$

\begin{tabular}{|rrrrl|}
\hline & total & centre & east lobe & west lobe \\
$\alpha_{\text {low }}$ & 0.77 & 0.72 & 0.82 & 0.72 \\
$\alpha_{\text {high }}$ & 1.38 & 1.64 & 1.40 & 0.89 \\
\hline
\end{tabular}

\section{5. $N G C 6251$}

At 2.7 and $4.8 \mathrm{GHz}$, there are no reliable flux densities available for the two lobes, nor for the entire source, as the maps had to be restricted in size at these frequencies. The spectral indices between $326 \mathrm{MHz}$ and $10.6 \mathrm{GHz}$ are $\alpha=0.77 \pm 0.04$ in the western lobe, and $\alpha=0.58 \pm 0.01$ for the entire source. In the case of the eastern lobe we can only determine a low-frequency spectral index of $\alpha=$ $0.90 \pm 0.07$. The core spectrum is almost straight, with a slight indication of a flattening towards high frequencies. The (straight) spectral index is $\alpha=0.40 \pm 0.01$. The hot spot spectra are straight within the errors, with a large difference in the spectral indices between the eastern $(\alpha=$ $1.41 \pm 0.13)$ and the western one $(\alpha=0.60 \pm 0.02)$. In the latter the influence of confusion is high because of the close neighbourhood of bright sources.

\section{Summary}

We have presented radio continuum maps of the five "classical" giant radio galaxies NGC 315, DA 240, 3C 236, 3C 326, and NGC 6251 at five different frequencies between $326 \mathrm{MHz}$ and $10.6 \mathrm{GHz}$. All observations were obtained with full polarization information. We have integrated the intensities of the entire sources and also within individual components to derive spectra of these areas. We have obtained reliable data across almost the entire radio spectrum for these complex radio galaxies. In general, a spectral steepening is found for the diffuse components, indicating particle ageing. Quantitative analyses will follow in forthcoming papers.

Acknowledgements. E. Fürst provided a modified version of the OSMOSE programme for single-horn observations which sets up the parameters required for CLEAN. M. Wieringa provided a copy of the REDUN task developed at Leiden University. KHM thanks the Deutsche Forschungsgemeinschaft for a doctoral fellowship. AGW thanks the National Sciences and Engineering Research Council of Canada for an operating grant while he was at Athabasca University. This work was supported by the Deutsche Forschungsgemeinschaft, grant KL533/4-2. This research has made use of the NASA/IPAC Extragalactic Data Base (NED) which is operated by the Jet Propulsion Laboratory, California Institute of Technology, under contract with the National Aeronautics and Space Administration. The Westerbork Radio Observatory is operated by the Netherlands Foundation for Research in Astronomy.

\section{References}

Baars J.W.M., Genzel R., Pauliny-Toth I.I.K., Witzel A., 1977, A\&A 61, 99 
Baker J.R., Preuss E., Whiteoak J.B., Zimmermann P., 1974, Nat 252, 552

Barthel R.D., Schilizzi R.T., Miley G.K., Jägers W.J., Strom R.G., 1985, A\&A 148, 243

Bridle A.H., Davis M.M., Fomalont E.B., Lequeux J., 1972, AJ 77,405

Bridle A.H., Davis M.M., Meloy D.A., Fomalont E.B., Strom R.G., Willis, A.G., 1976, Nat 262, 179

Bridle A.H., Davis M.M., Fomalont E.B., W illis A.G., Strom R.G., 1979, ApJ 228, L9

Emerson D.T., Gräve R., 1988, A\&A 190, 353

Jägers W.J., 1986, Ph. D. thesis, University of Leiden

Jägers W.J., 1987a, A\&AS 71, 75

Jägers W.J., 1987b, A\&AS 71, 603

Klein U., Mack K.-H., 1995, in: Emerson D.T. (ed.) Multi-Feed Systems for Radio Telescopes, ASP Conf. Ser. 75, 318

Klein U., Mack K.-H., Strom R., Wielebinski R., Achatz U., 1994, A\&A 283, 729

Mackay C.D., 1969, MNRAS 145, 31

Perley R.A., 1986, in: Perley R.A, Schwab F.R., Bridle A.H. (ed.) Synthesis Imaging, NRAO, ch. 11

Perley R.A., Bridle A.H., Willis A.G., 1984, ApJS 54, 291

Saripalli L., Mack K.-H., Klein U., Strom R., Singal A.K., 1996,
A\&A 306, 708

Saunders R., Baldwin J.E., Pooley G.G., Warner P.J., 1981, MNRAS 197, 287

Spoelstra T.A.Th., 1981, NFRA Internal Technical Report 162

Stoffel H., Wielebinski R., 1978, A\&A 68, 307

Strom R.G., Willis A.G., 1980, A\&A 85, 36

Strom R.G., Baker J.R., Willis A.G., 1981, A\&A 100, 220

Tsien S.C., 1982, MNRAS 200, 377

Waggett P.C., Warner P.J., Baldwin J.E., 1977, MNRAS 181, 465

Wardle J.F.C., Kronberg P.P., 1974, ApJ 194, 249

Willis A.G., O'Dea C.P., 1990, in: Beck R., Kronberg P.P., Wielebinski R. (eds.) Proc. IAU Symp. 140, Galactic and Intergalactic Magnetic Fields. Kluwer, Dordrecht, p. 455

Willis A.G., Strom R.G., 1978, A\&A 62, 375

Willis A.G., Strom R.G., Wilson A.S., 1974, Nat 250, 625

Willis A.G., Strom R.G., Bridle A.H., Fomalont E.B., 1981, A\&A 95, 250

Willis A.G., Strom R.G., Perley R.A., Bridle A.H., 1982, in: Heeschen D.S., Wade C.M. (eds.) Proc. IAU Symp. 97, Extragalactic Radio Sources. Reidel, Dordrecht, p. 141

Willis A.G., Wilson A.S., Strom R.G., 1978, A\&A 66, L1 


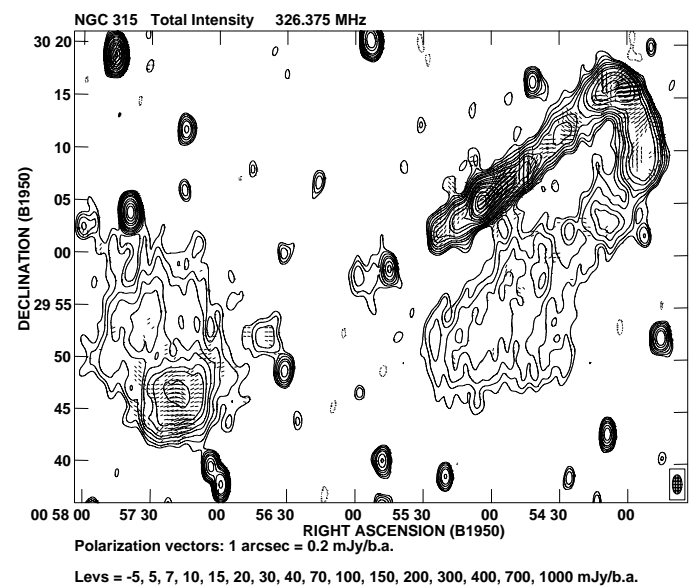

Fig. 1. Map of the total intensity of NGC 315 at $\lambda 92 \mathrm{~cm}$. Also shown are the $\boldsymbol{E}$-vectors of the linearly polarized emission. Their lengths are proportional to the polarized intensity

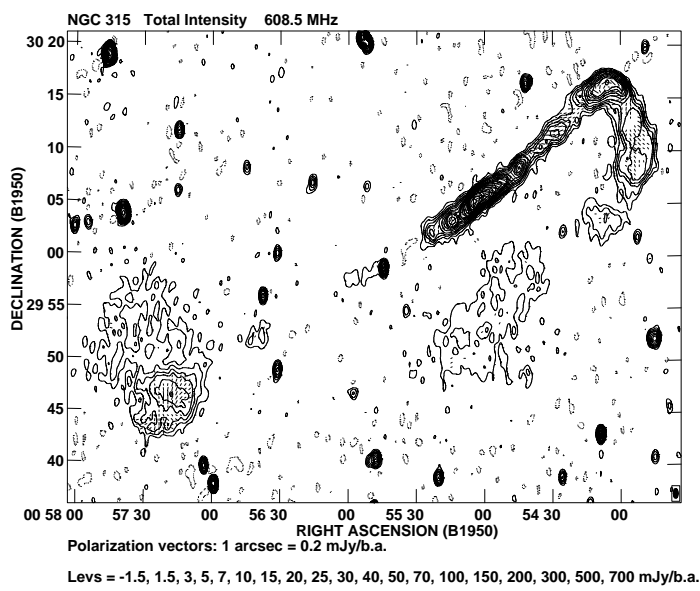

Fig. 3. Map of the total intensity of NGC 315 at $\lambda 49 \mathrm{~cm}$ (same layout as in Fig. 1)

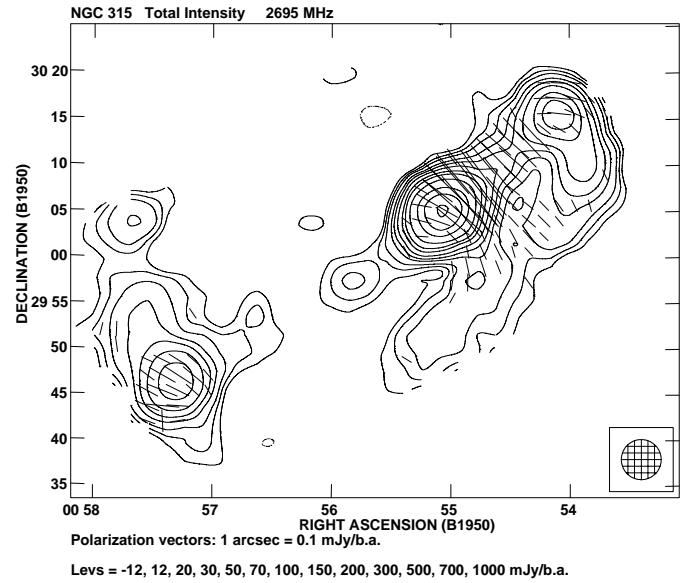

Fig. 5. Map of the total intensity of NGC 315 at $\lambda 11 \mathrm{~cm}$ (same layout as in Fig. 1)

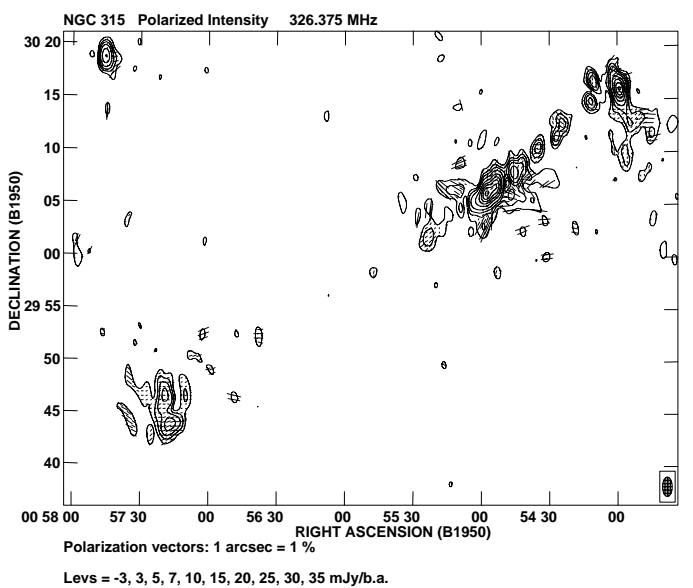

Fig. 2. Map of the linearly polarized intensity of NGC 315 at $\lambda 92 \mathrm{~cm}$. The $\boldsymbol{E}$-vectors have lengths proportional to the fractional polarization

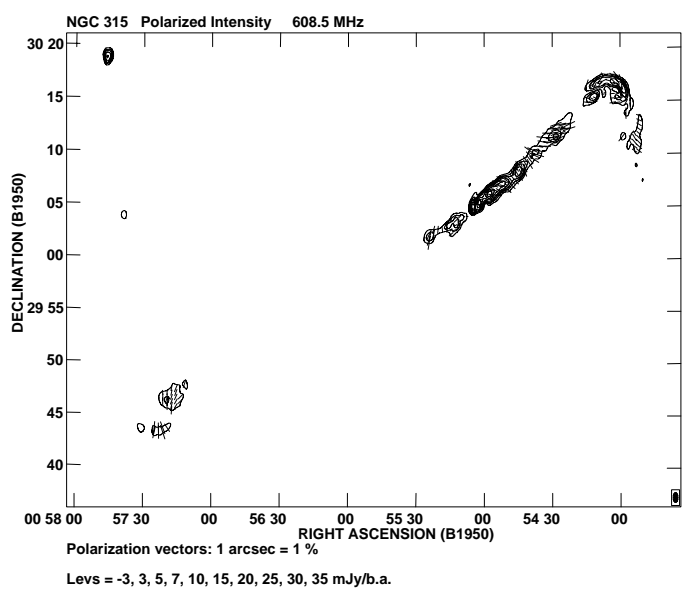

Fig. 4. Map of the linearly polarized intensity of NGC 315 at $\lambda 49 \mathrm{~cm}$ (same layout as in Fig. 2)

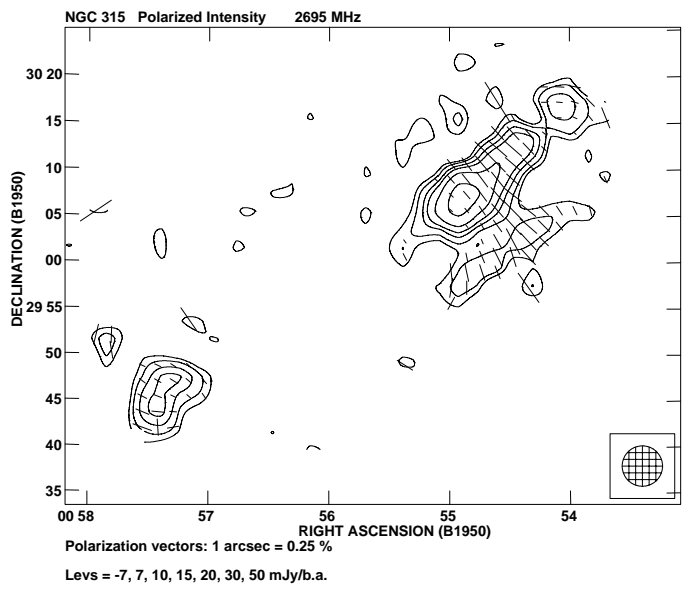

Fig. 6. Map of the linearly polarized intensity of NGC 315 at $\lambda 11 \mathrm{~cm}$ (same layout as in Fig. 2) 


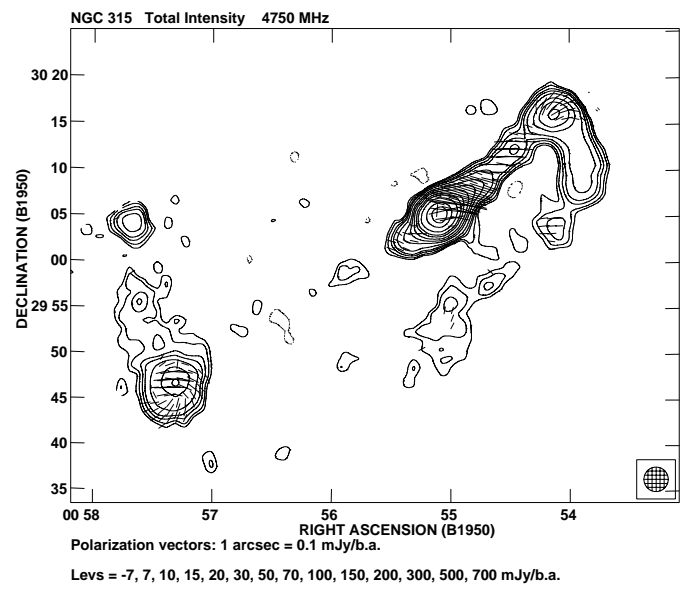

Fig. 7. Map of the total intensity of NGC 315 at $\lambda 6.3 \mathrm{~cm}$ (same layout as in Fig. 1)

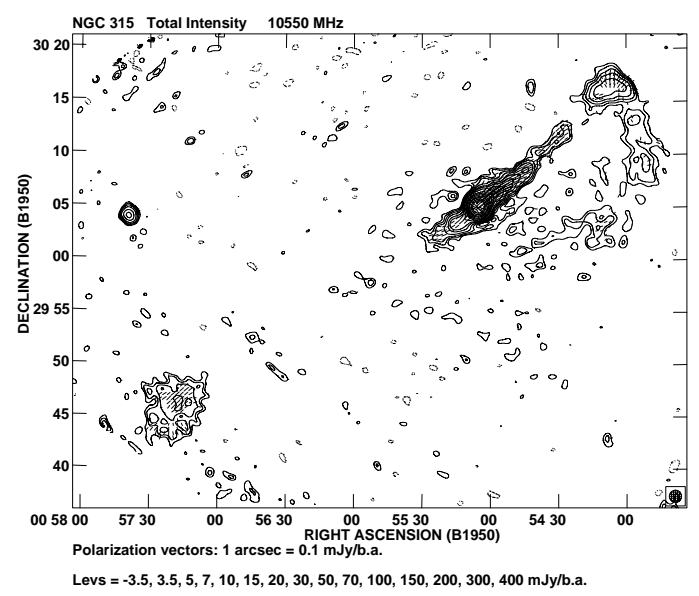

Fig. 9. Map of the total intensity of NGC 315 at $\lambda 2.8 \mathrm{~cm}$ (same layout as in Fig. 1)

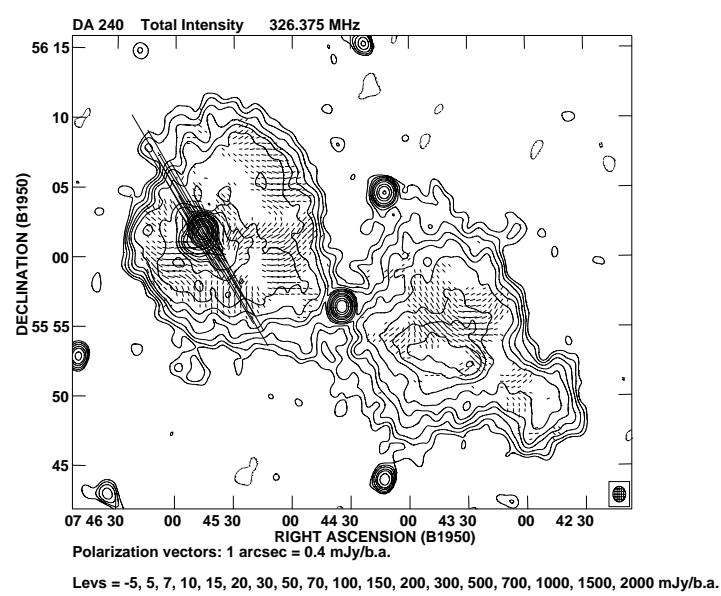

Fig. 11. Map of the total intensity of DA 240 at $\lambda 92 \mathrm{~cm}$ (same layout as in Fig. 1)

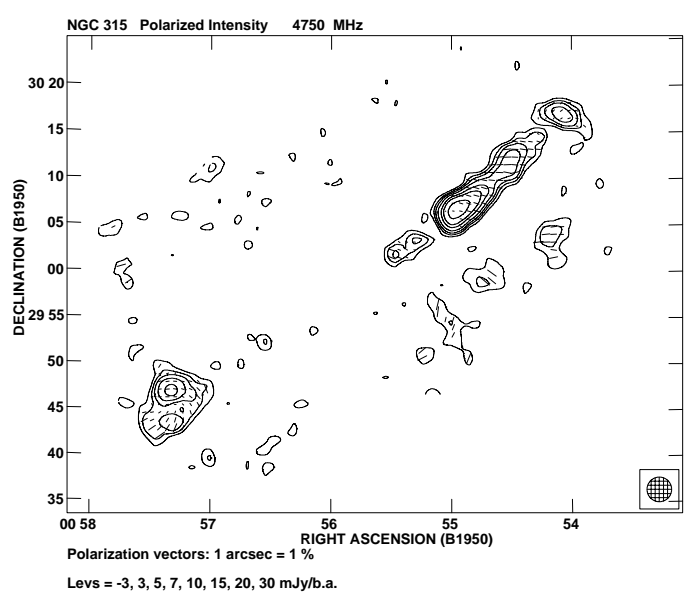

Fig. 8. Map of the linearly polarized intensity of NGC 315 at $\lambda 6.3 \mathrm{~cm}$ (same layout as in Fig. 2)

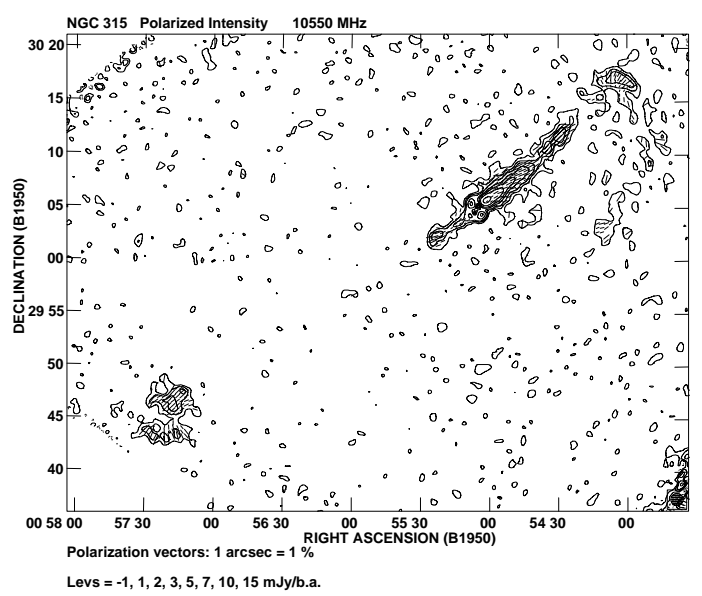

Fig. 10. Map of the linearly polarized intensity of NGC 315 at $\lambda 2.8 \mathrm{~cm}$ (same layout as in Fig. 2)

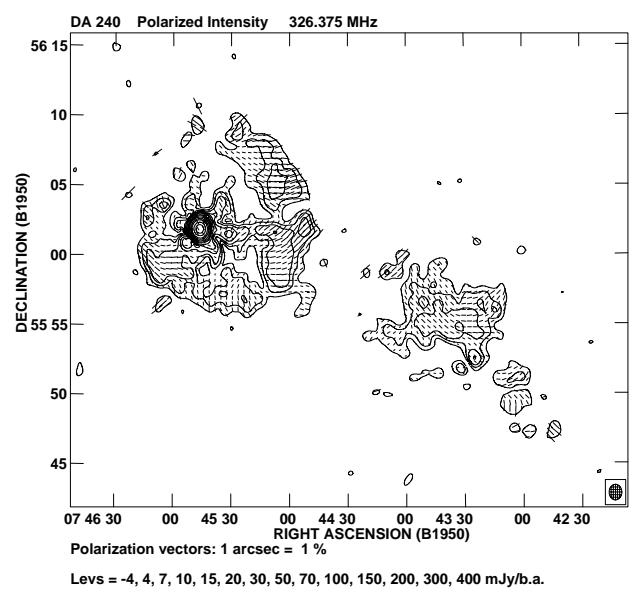

Fig. 12. Map of the linearly polarized intensity of DA 240 at $\lambda 92 \mathrm{~cm}$ (same layout as in Fig. 2) 


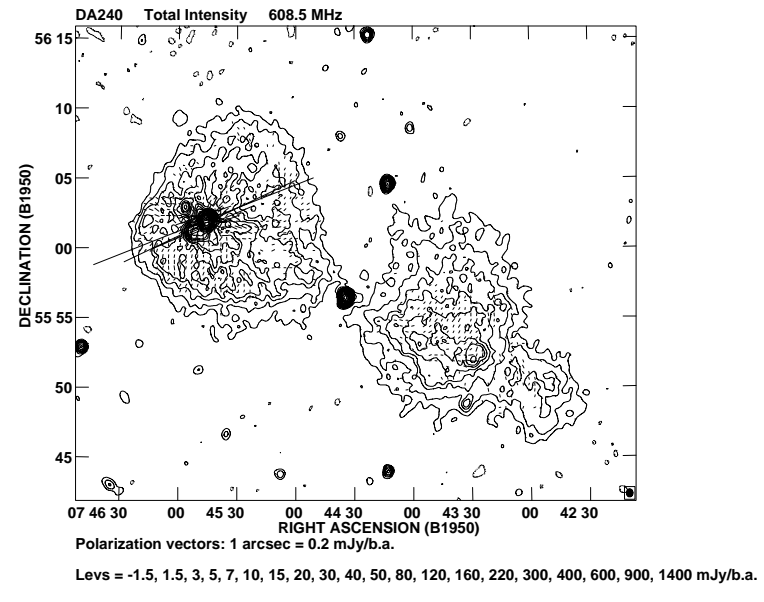

Fig. 13. Map of the total intensity of DA 240 at $\lambda 49 \mathrm{~cm}$ (same layout as in Fig. 1)

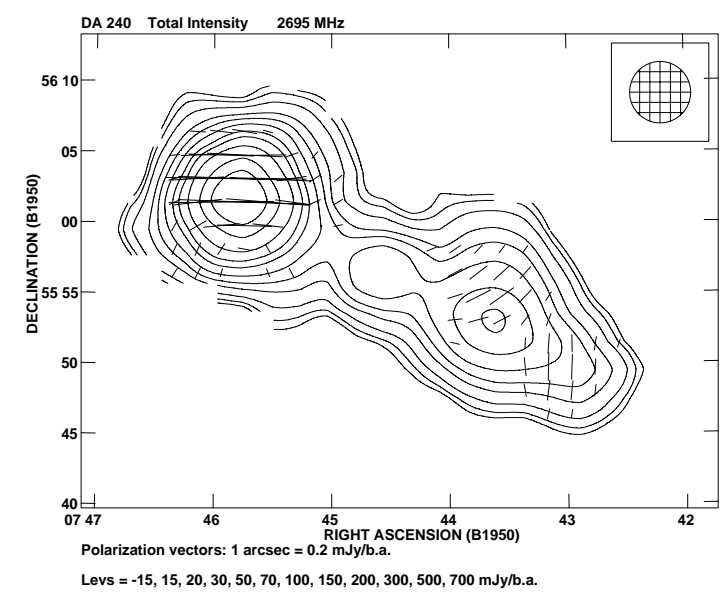

Fig. 15. Map of the total intensity of DA 240 at $\lambda 11 \mathrm{~cm}$ (same layout as in Fig. 1)

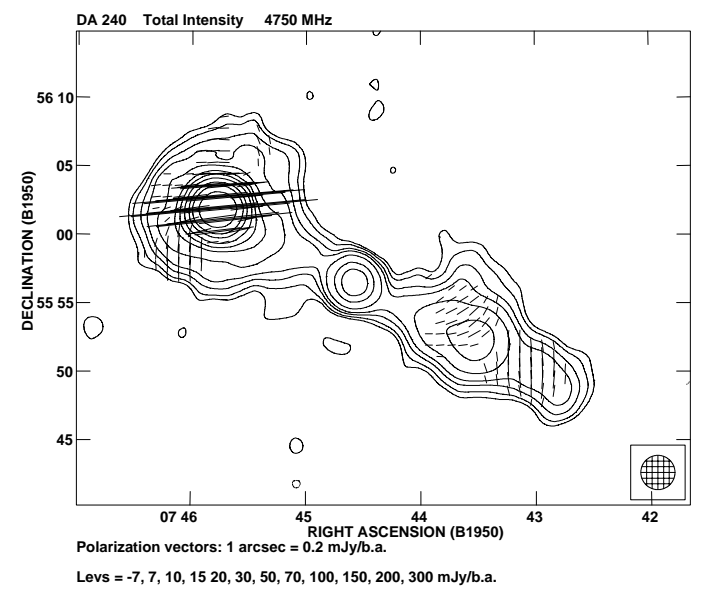

Fig. 17. Map of the total intensity of DA 240 at $\lambda 6.3 \mathrm{~cm}$ (same layout as in Fig. 1)

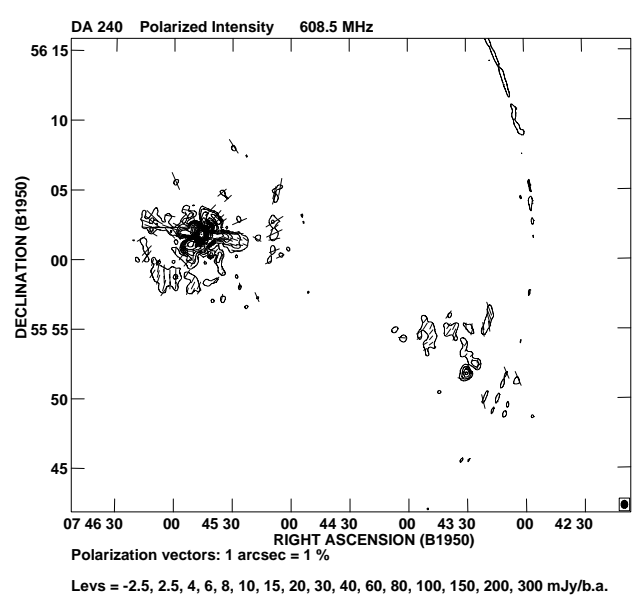

Fig. 14. Map of the linearly polarized intensity of DA 240 at $\lambda 49 \mathrm{~cm}$ (same layout as in Fig. 2)

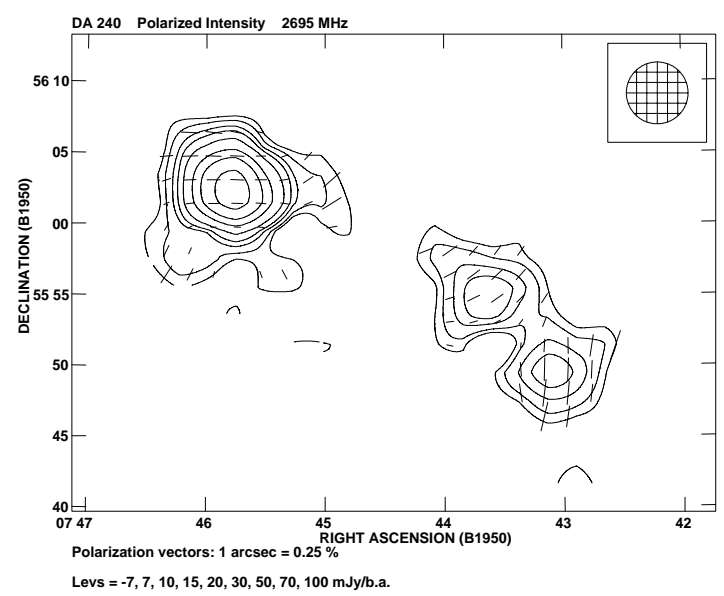

Fig. 16. Map of the linearly polarized intensity of DA 240 at $\lambda 11 \mathrm{~cm}$ (same layout as in Fig. 2)

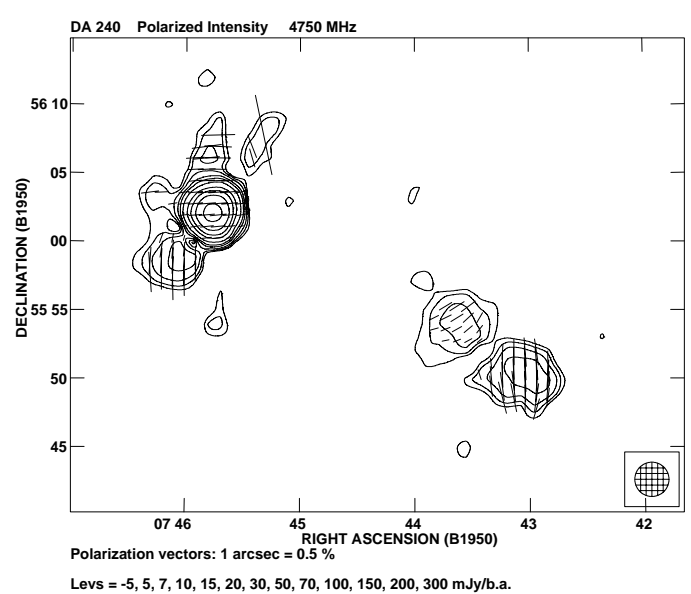

Fig. 18. Map of the linearly polarized intensity of DA 240 at $\lambda 6.3 \mathrm{~cm}$ (same layout as in Fig. 2) 


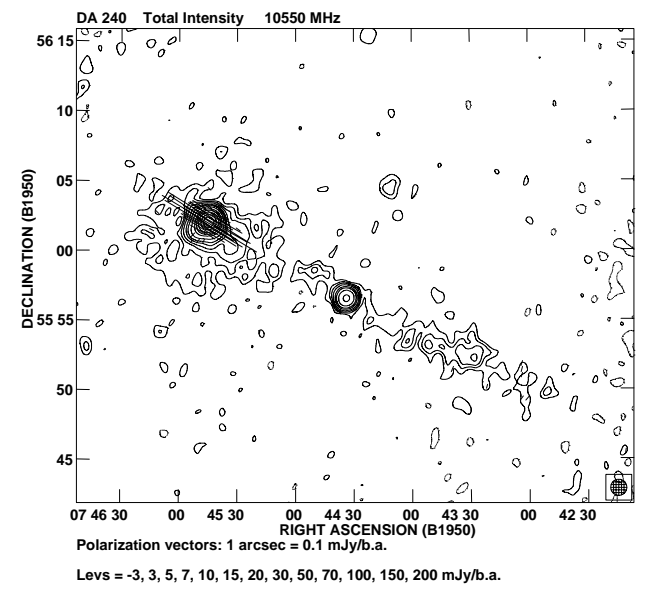

Fig. 19. Map of the total intensity of DA 240 at $\lambda 2.8 \mathrm{~cm}$ (same layout as in Fig. 1)

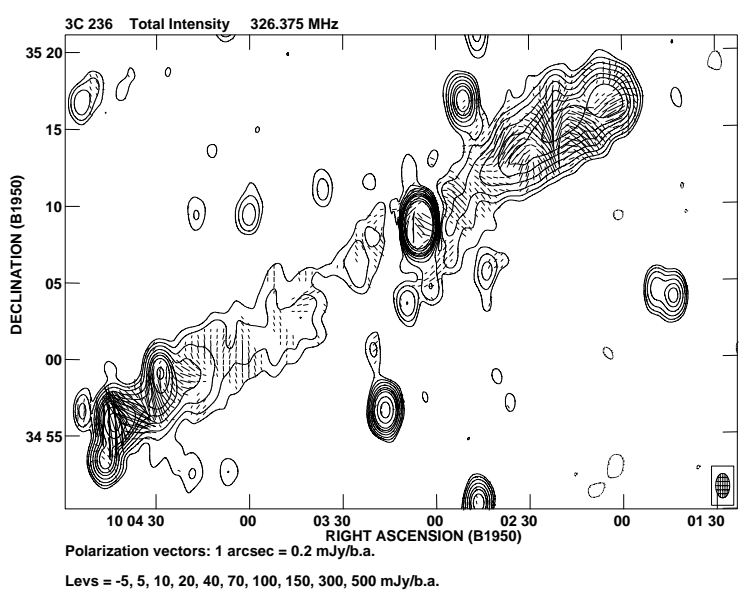

Fig. 21. Map of the total intensity of $3 \mathrm{C} 236$ at $\lambda 92 \mathrm{~cm}$ (same layout as in Fig. 1)

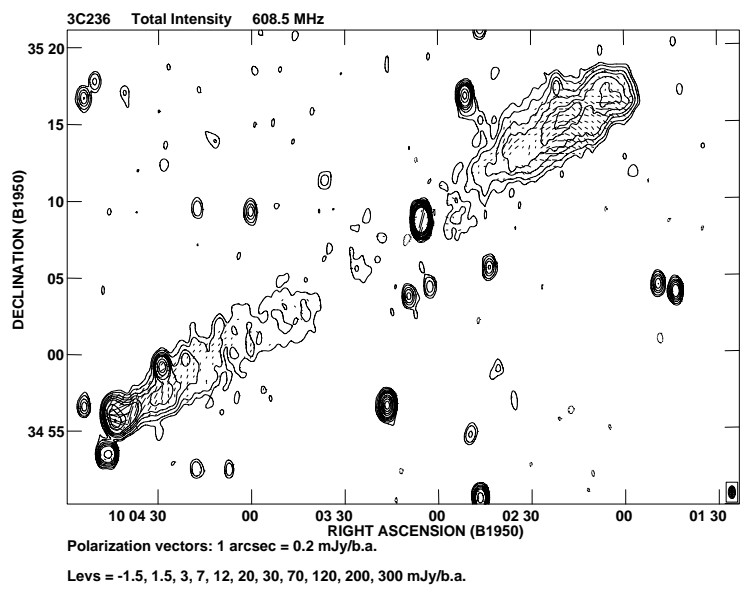

Fig. 23. Map of the total intensity of $3 \mathrm{C} 236$ at $\lambda 49 \mathrm{~cm}$ (same layout as in Fig. 1)

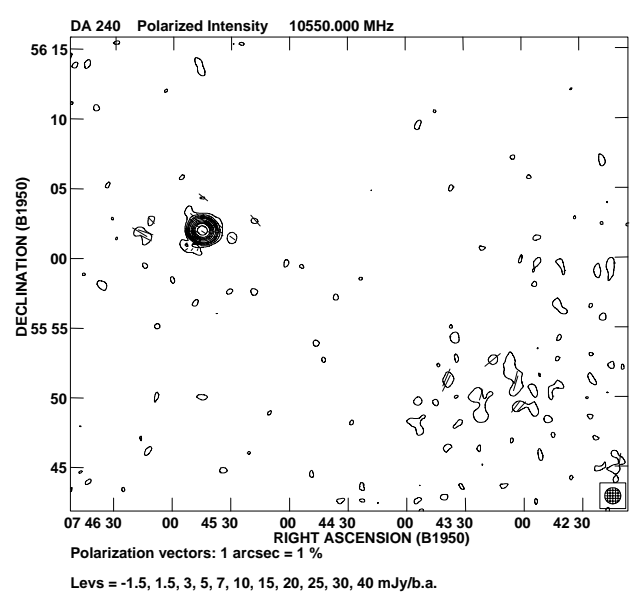

Fig. 20. Map of the linearly polarized intensity of DA 240 at $\lambda 2.8 \mathrm{~cm}$ (same layout as in Fig. 2)

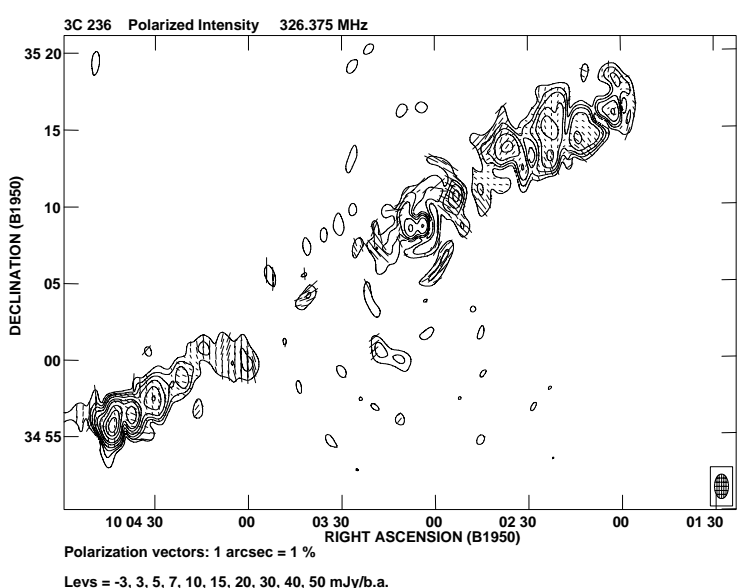

Fig. 22. Map of the linearly polarized intensity of $3 \mathrm{C} 236$ at $\lambda 92 \mathrm{~cm}$ (same layout as in Fig. 2)

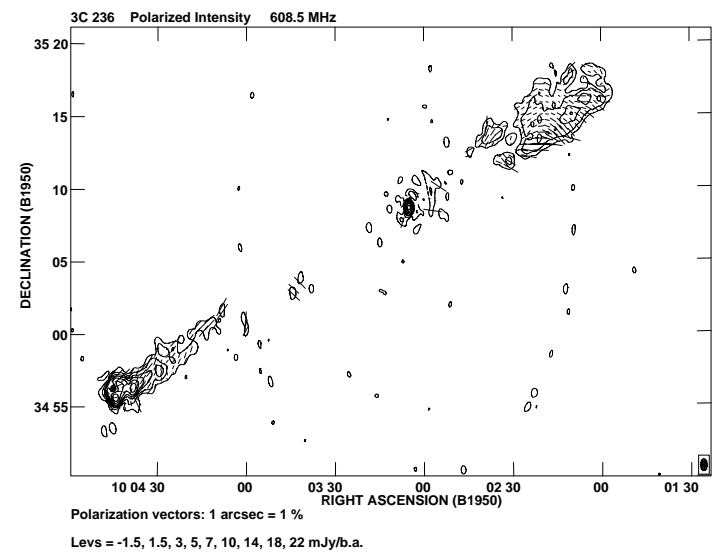

Fig. 24. Map of the linearly polarized intensity of $3 \mathrm{C} 236$ at $\lambda 49 \mathrm{~cm}$ (same layout as in Fig. 2) 


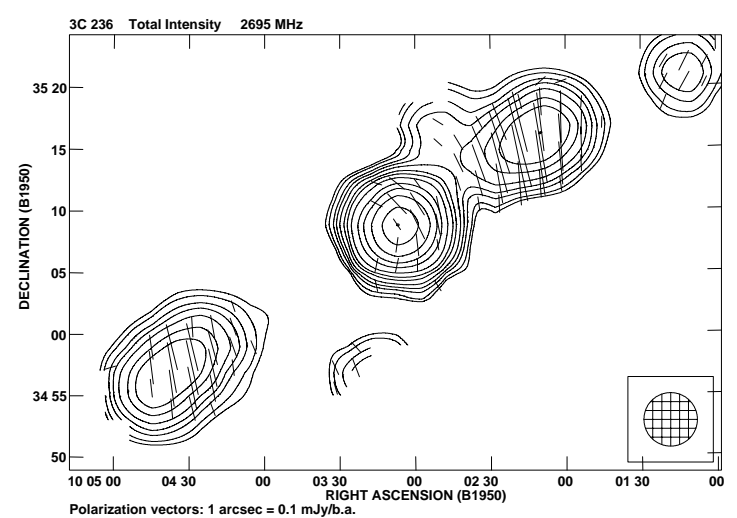

Levs $=-20,20,30,50,70,100,150,200,300,500,700,1000,1500,2000$ mJy/b.a.

Fig. 25. Map of the total intensity of $3 \mathrm{C} 236$ at $\lambda 11 \mathrm{~cm}$ (same layout as in Fig. 1)

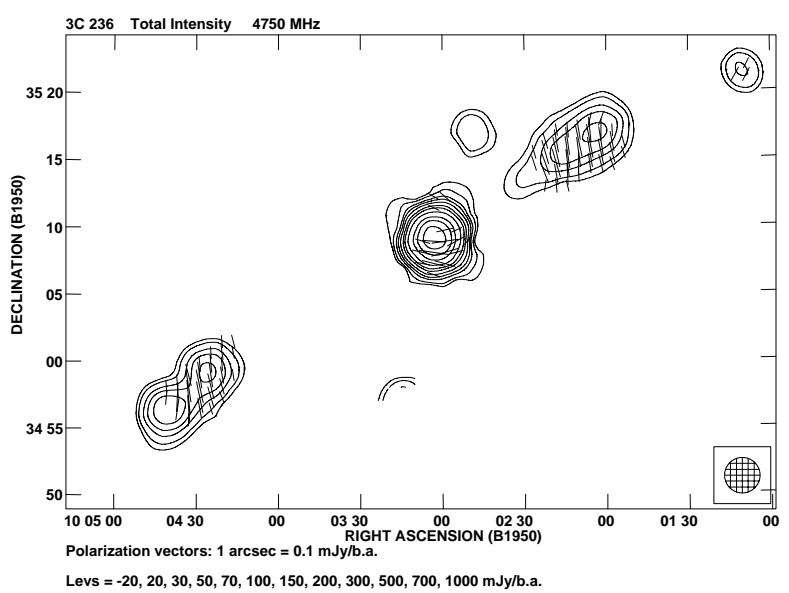

Fig. 27. Map of the total intensity of $3 \mathrm{C} 236$ at $\lambda 6.3 \mathrm{~cm}$ (same layout as in Fig. 1)

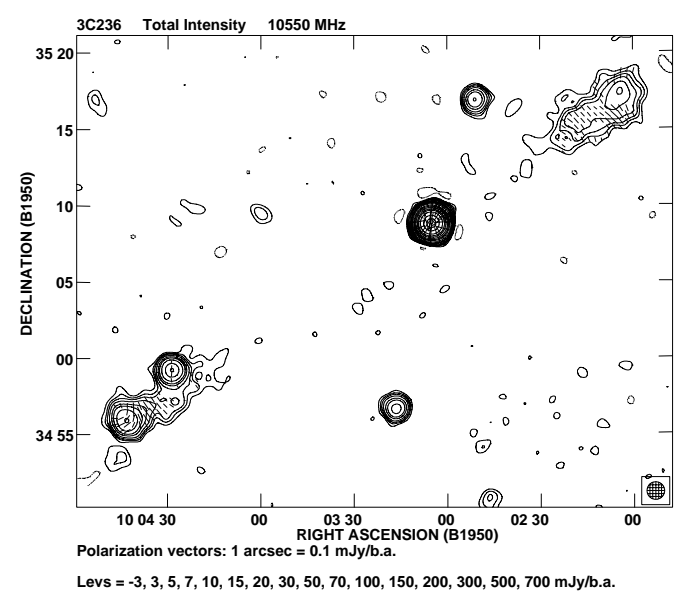

Fig. 29. Map of the total intensity of $3 \mathrm{C} 236$ at $\lambda 2.8 \mathrm{~cm}$ (same layout as in Fig. 1)

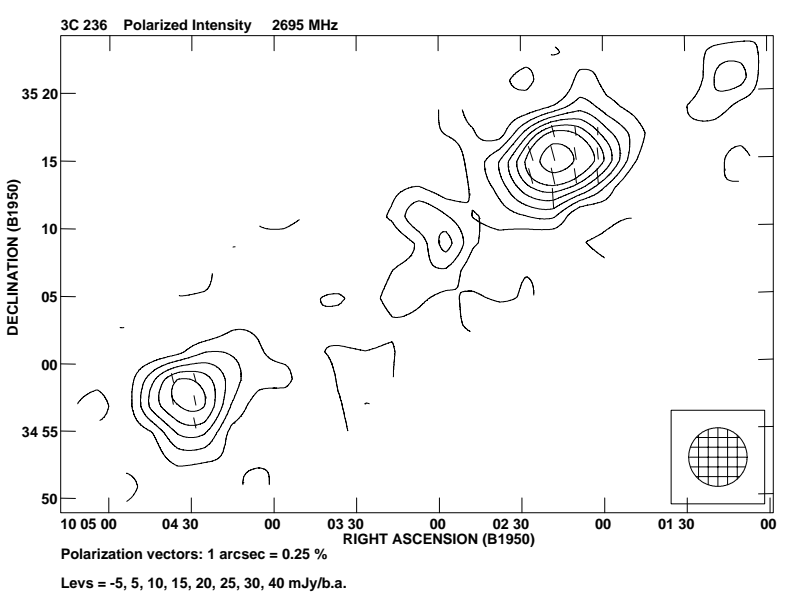

Fig. 26. Map of the linearly polarized intensity of $3 \mathrm{C} 236$ at $\lambda 11 \mathrm{~cm}$ (same layout as in Fig. 2)

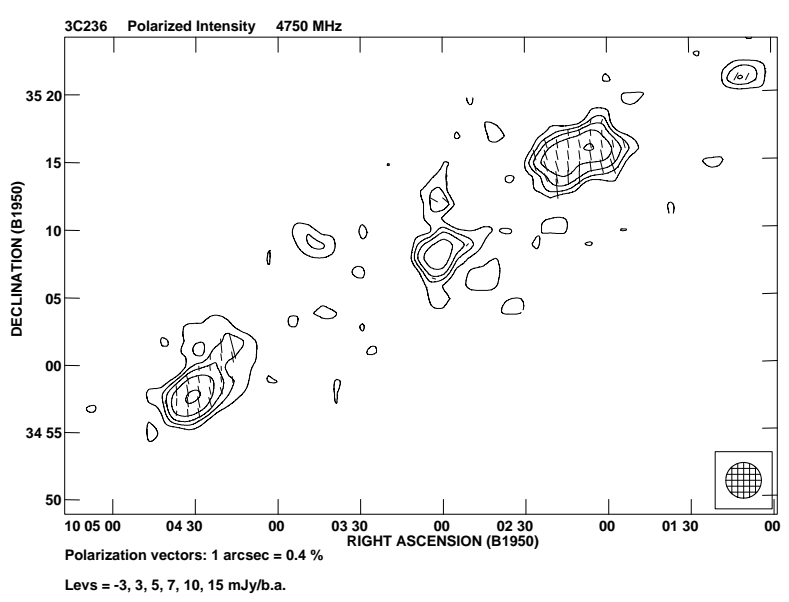

Fig. 28. Map of the linearly polarized intensity of $3 \mathrm{C} 236$ at $\lambda 6.3 \mathrm{~cm}$ (same layout as in Fig. 2)

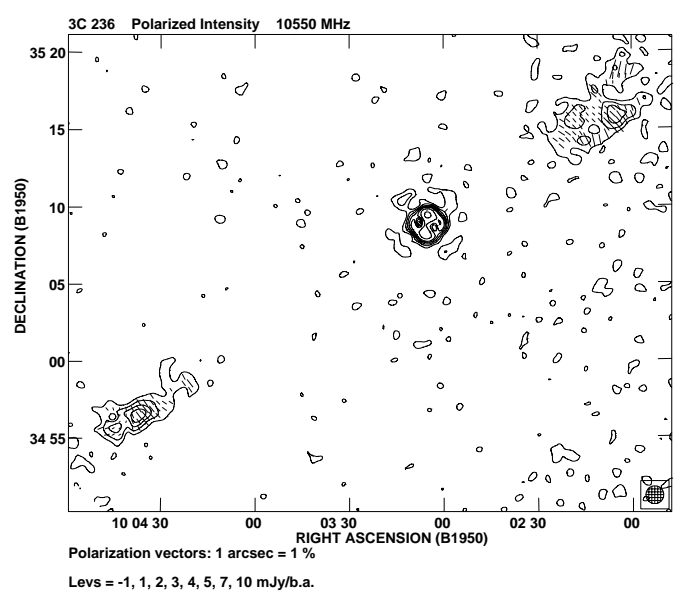

Fig. 30. Map of the linearly polarized intensity of $3 \mathrm{C} 236$ at $\lambda 2.8 \mathrm{~cm}$ (same layout as in Fig. 2) 


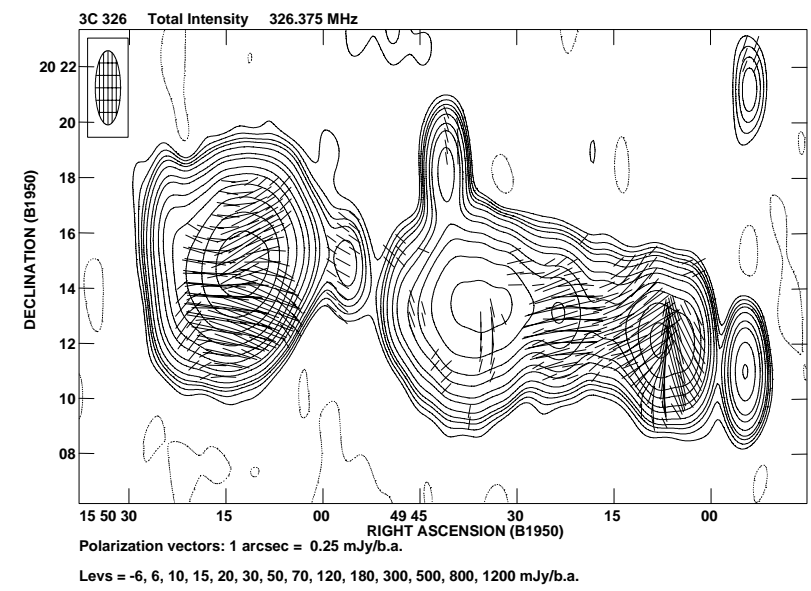

Fig. 31. Map of the total intensity of $3 \mathrm{C} 326$ at $\lambda 92 \mathrm{~cm}$ (same layout as in Fig. 1)

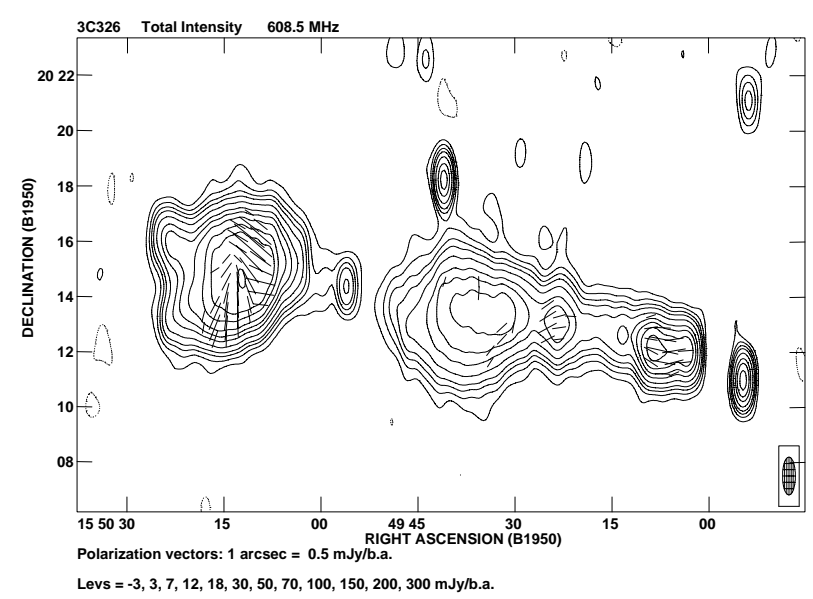

Fig. 33. Map of the total intensity of $3 \mathrm{C} 326$ at $\lambda 49 \mathrm{~cm}$ (same layout as in Fig. 1)

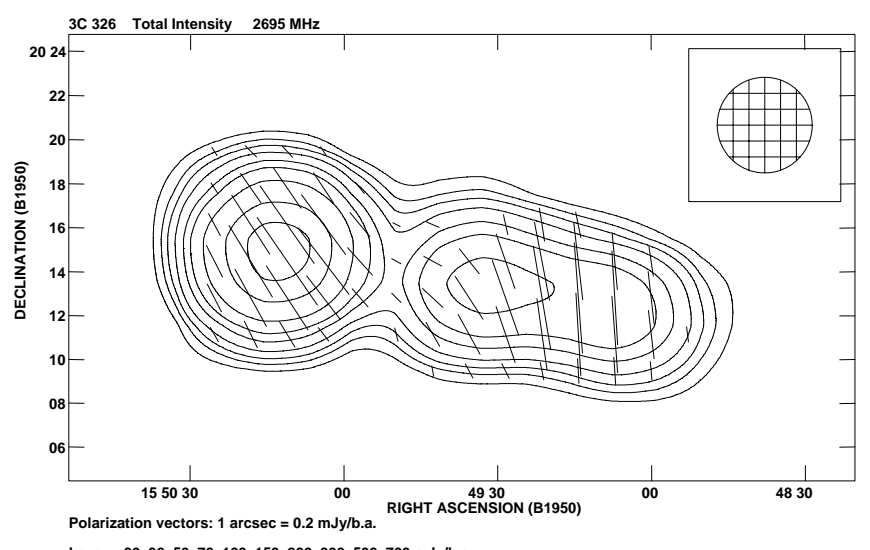

Fig. 35. Map of the total intensity of $3 \mathrm{C} 326$ at $\lambda 11 \mathrm{~cm}$ (same layout as in Fig. 1)

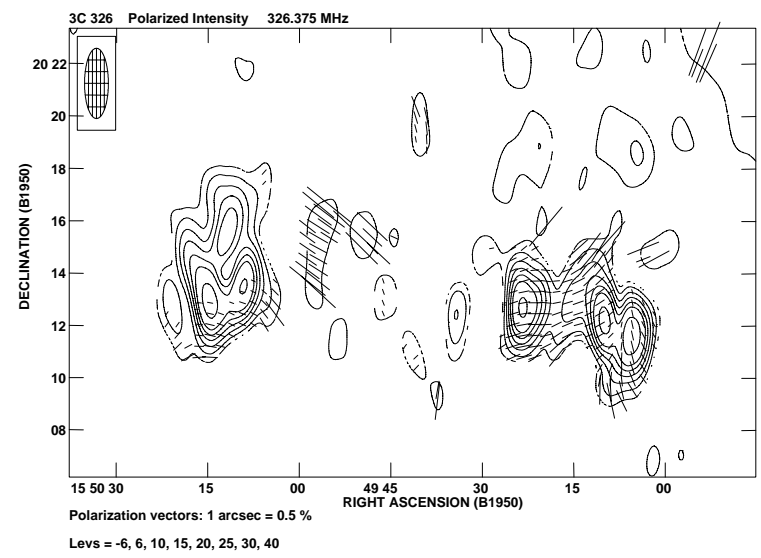

Fig. 32. Map of the linearly polarized intensity of $3 \mathrm{C} 326$ at $\lambda 92 \mathrm{~cm}$ (same layout as in Fig. 2)

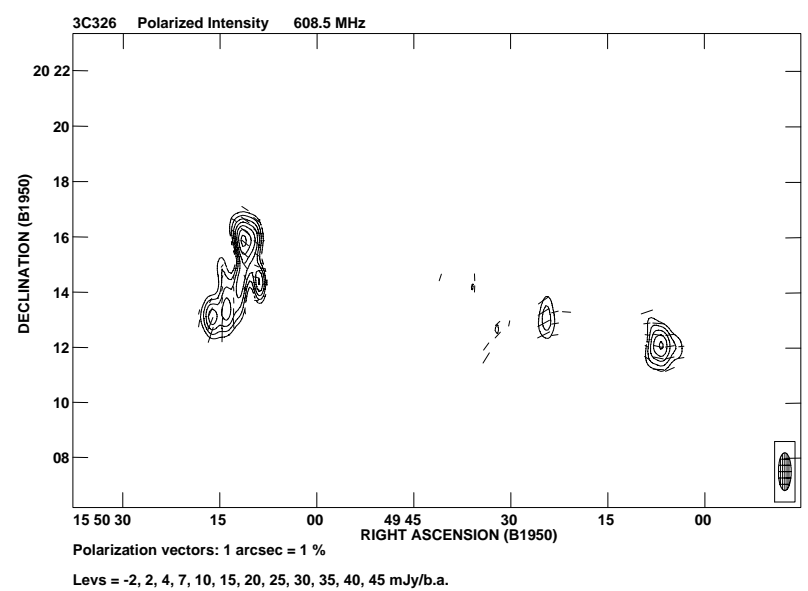

Fig. 34. Map of the linearly polarized intensity of 3C 326 at $\lambda 49 \mathrm{~cm}$ (same layout as in Fig. 2)

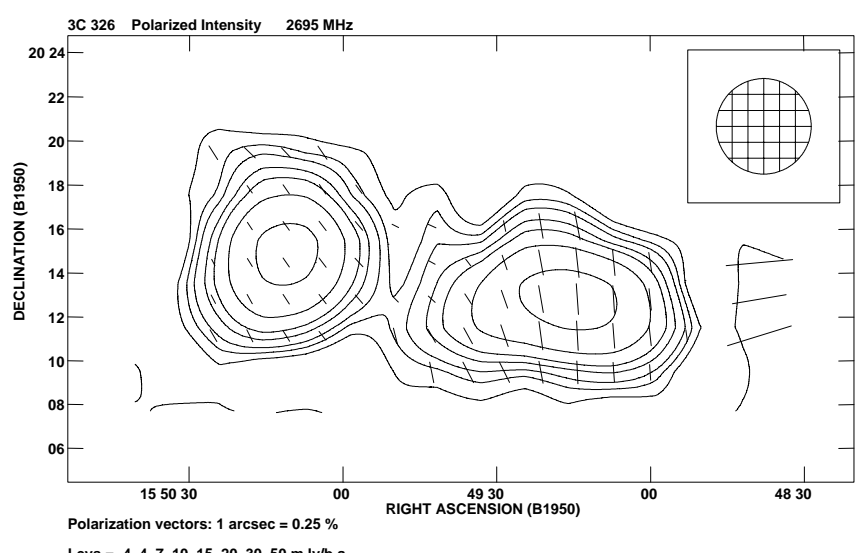

Fig. 36. Map of the linearly polarized intensity of 3C 326 at $\lambda 11 \mathrm{~cm}$ (same layout as in Fig. 2) 


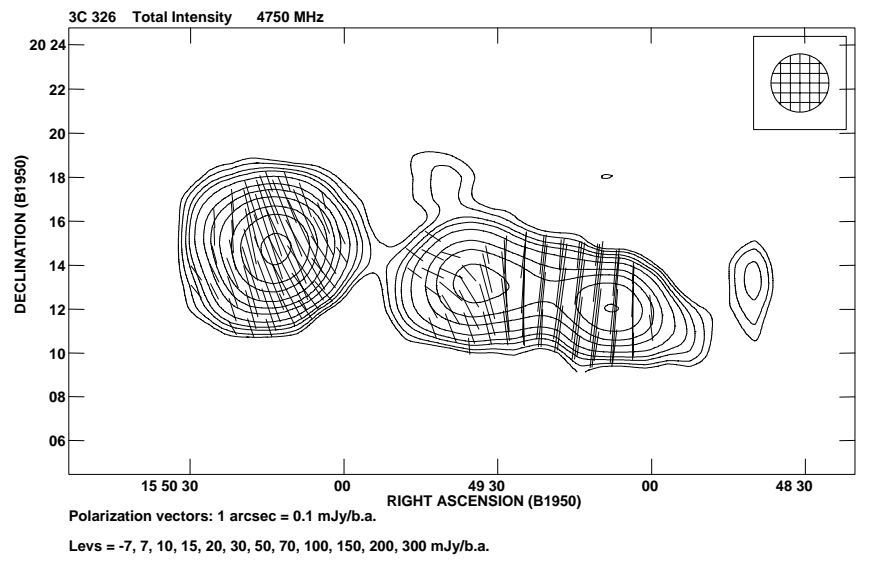

Fig. 37. Map of the total intensity of $3 \mathrm{C} 326$ at $\lambda 6.3 \mathrm{~cm}$ (same layout as in Fig. 1)

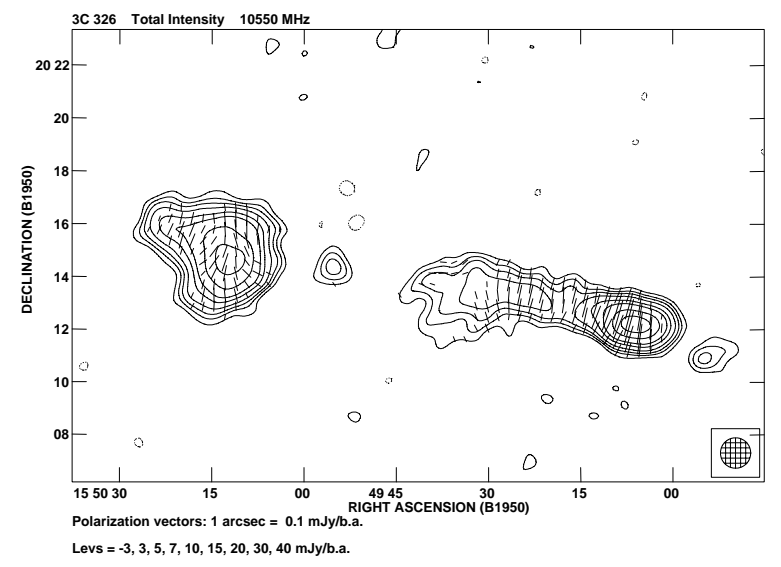

Fig. 39. Map of the total intensity of $3 \mathrm{C} 326$ at $\lambda 2.8 \mathrm{~cm}$ (same layout as in Fig. 1)

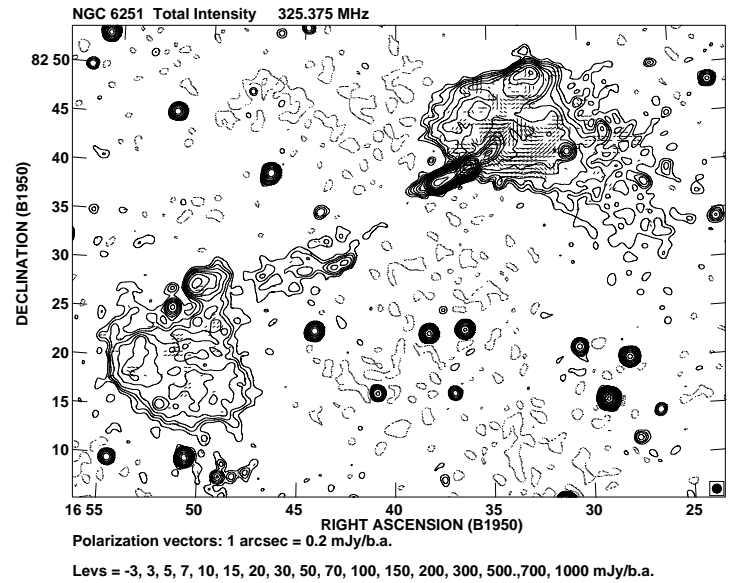

Fig. 41. Map of the total intensity of NGC 6251 at $\lambda 92 \mathrm{~cm}$ (same layout as in Fig. 1)

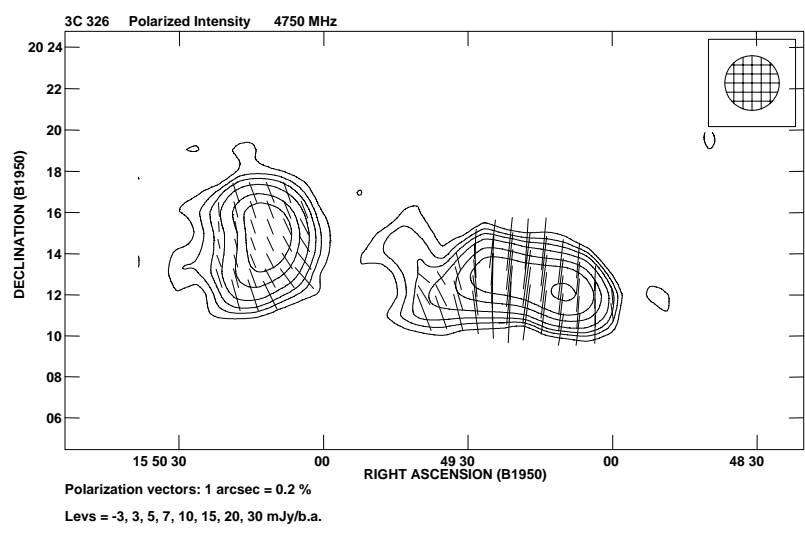

Fig. 38. Map of the linearly polarized intensity of $3 \mathrm{C} 326$ at $\lambda 6.3 \mathrm{~cm}$ (same layout as in Fig. 2)

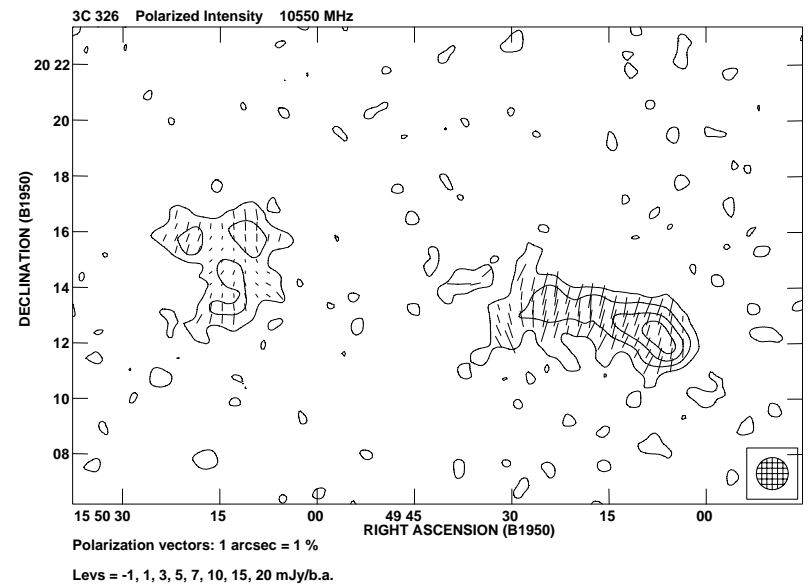

Fig. 40. Map of the linearly polarized intensity of $3 \mathrm{C} 326$ at $\lambda 2.8 \mathrm{~cm}$ (same layout as in Fig. 2)

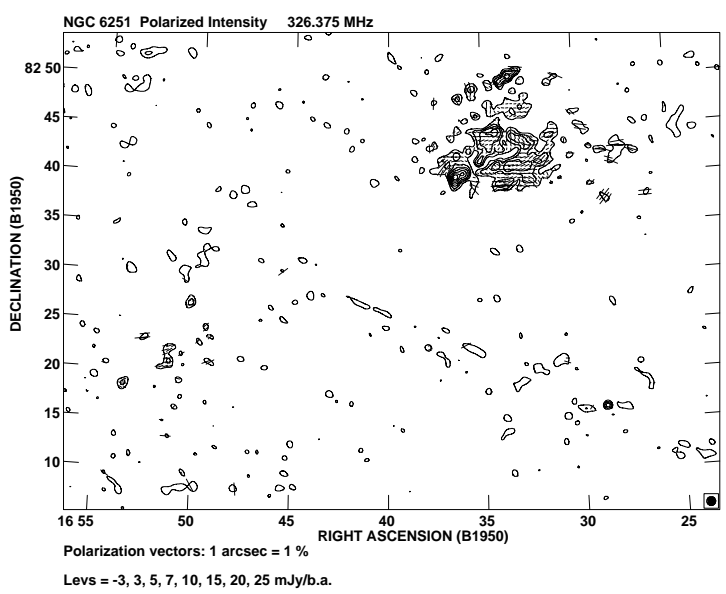

Fig. 42. Map of the linearly polarized intensity of NGC 6251 at $\lambda 92 \mathrm{~cm}$ (same layout as in Fig. 2) 


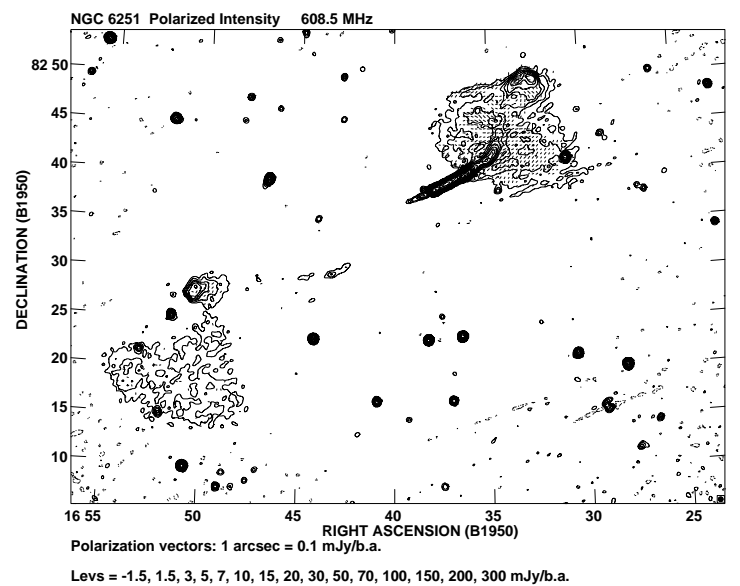

Fig. 43. Map of the total intensity of NGC 6251 at $\lambda 49 \mathrm{~cm}$ (same layout as in Fig. 1)

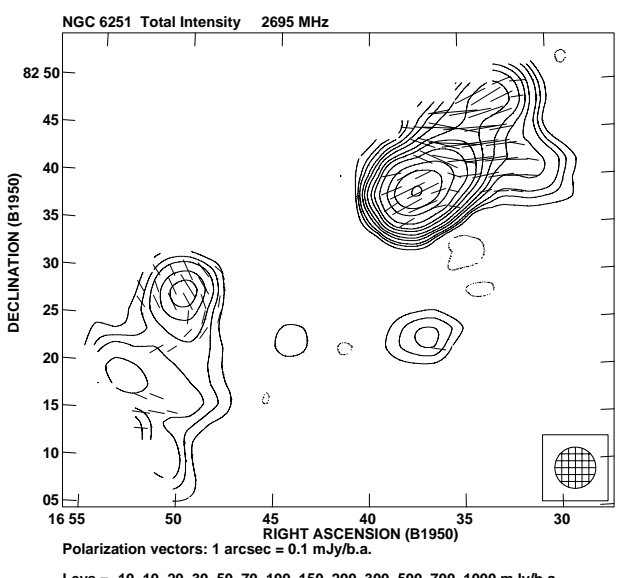

Fig. 45. Map of the total intensity of NGC 6251 at $\lambda 11 \mathrm{~cm}$ (same layout as in Fig. 1)

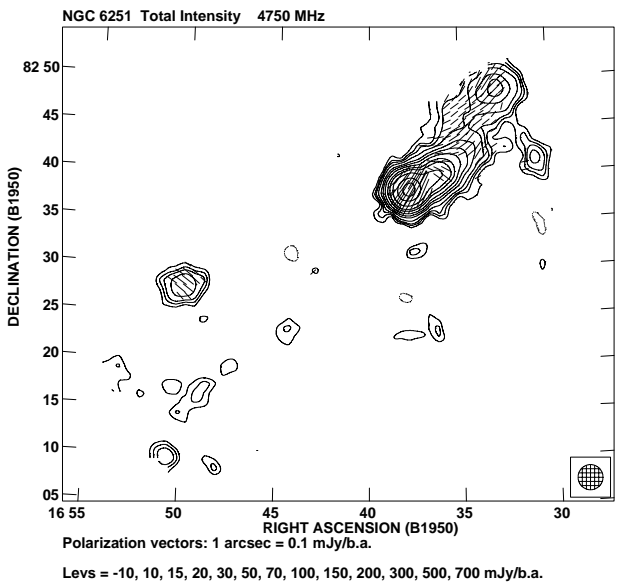

Fig. 47. Map of the total intensity of NGC 6251 at $\lambda 6.3 \mathrm{~cm}$ (same layout as in Fig. 1)

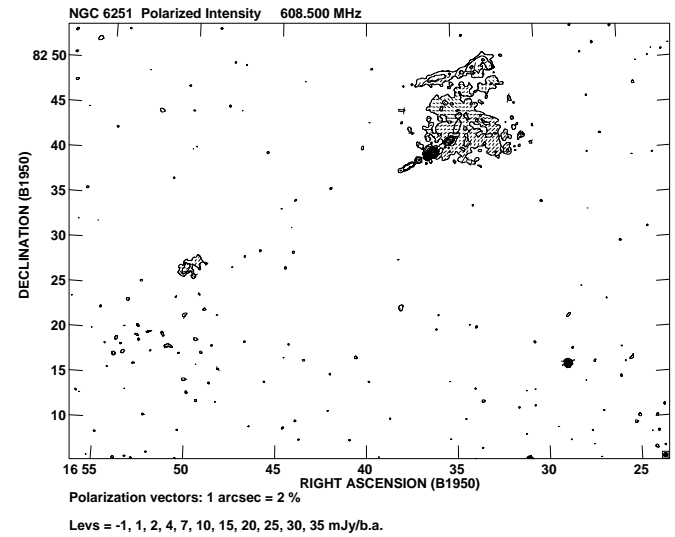

Fig. 44. Map of the linearly polarized intensity of NGC 6251 at $\lambda 49 \mathrm{~cm}$ (same layout as in Fig. 2)

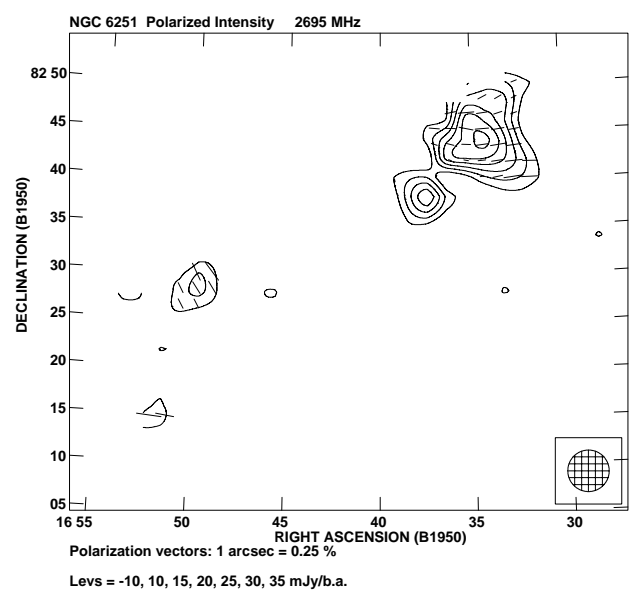

Fig. 46. Map of the linearly polarized intensity of NGC 6251 at $\lambda 11 \mathrm{~cm}$ (same layout as in Fig. 2)

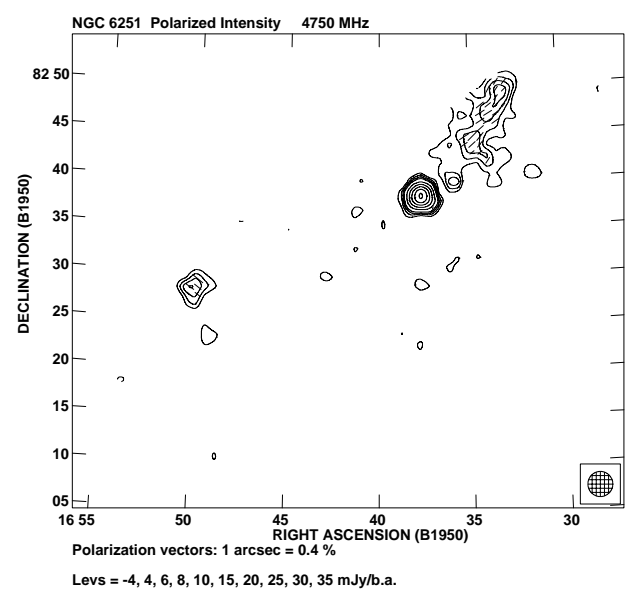

Fig. 48. Map of the linearly polarized intensity of NGC 6251 at $\lambda 6.3 \mathrm{~cm}$ (same layout as in Fig. 2) 

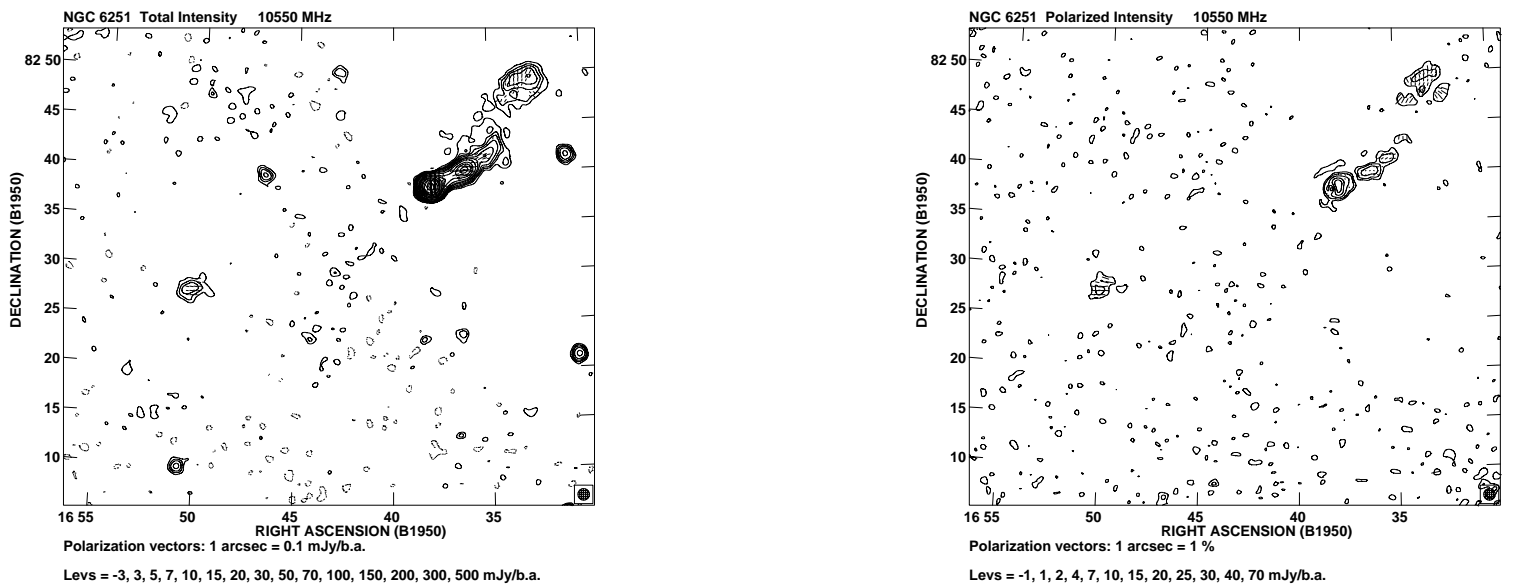

Fig. 49. Map of the total intensity of NGC 6251 at $\lambda 2.8 \mathrm{~cm}$ (same layout as in Fig. 1)

Fig. 50. Map of the linearly polarized intensity of NGC 6251 at $\lambda 2.8 \mathrm{~cm}$ (same layout as in Fig. 2) 

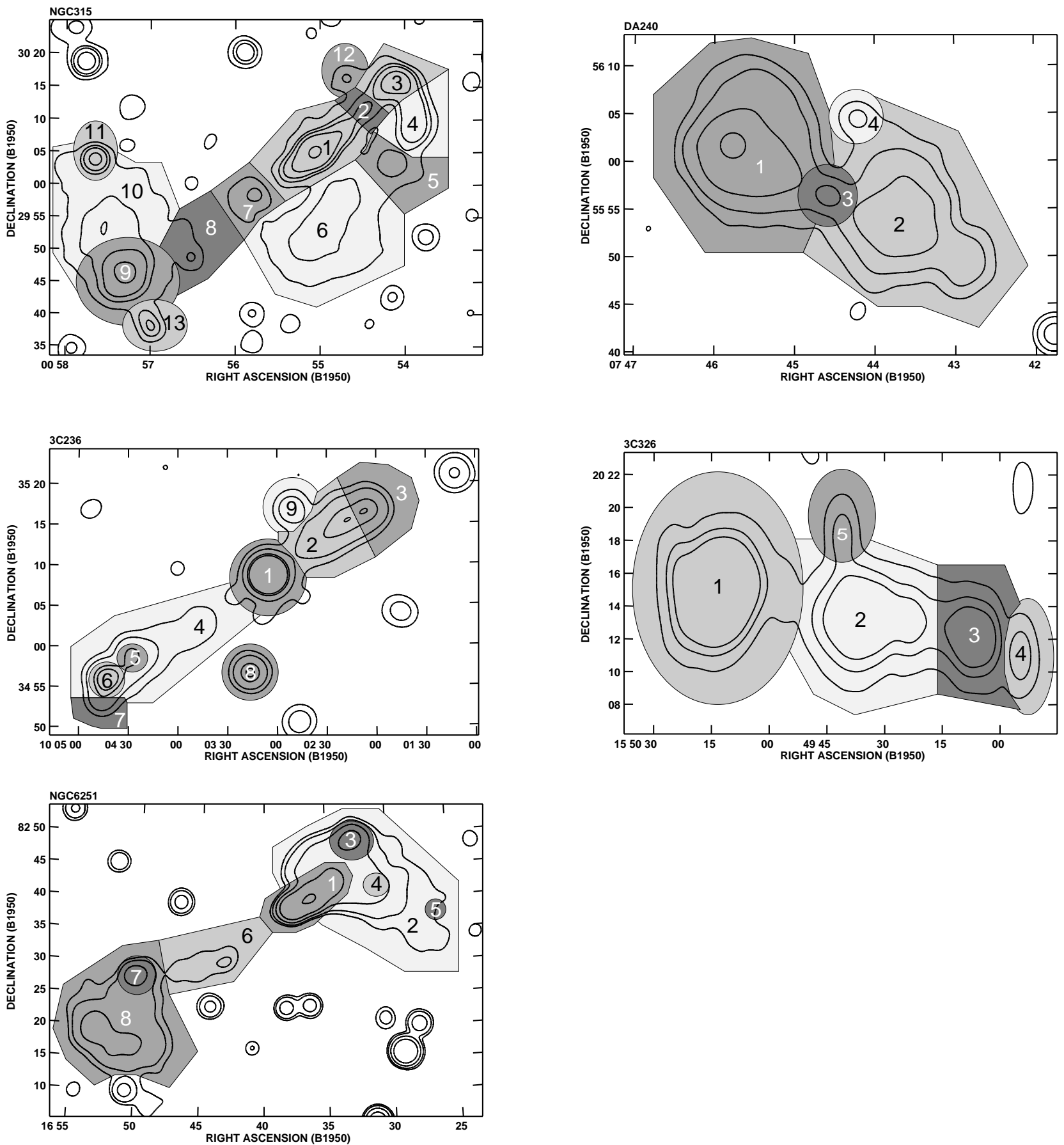

Fig. 51. "Finding charts" of GRGs, delineating the individual regions where integrated flux densities have been determined (Tables 7-11). 

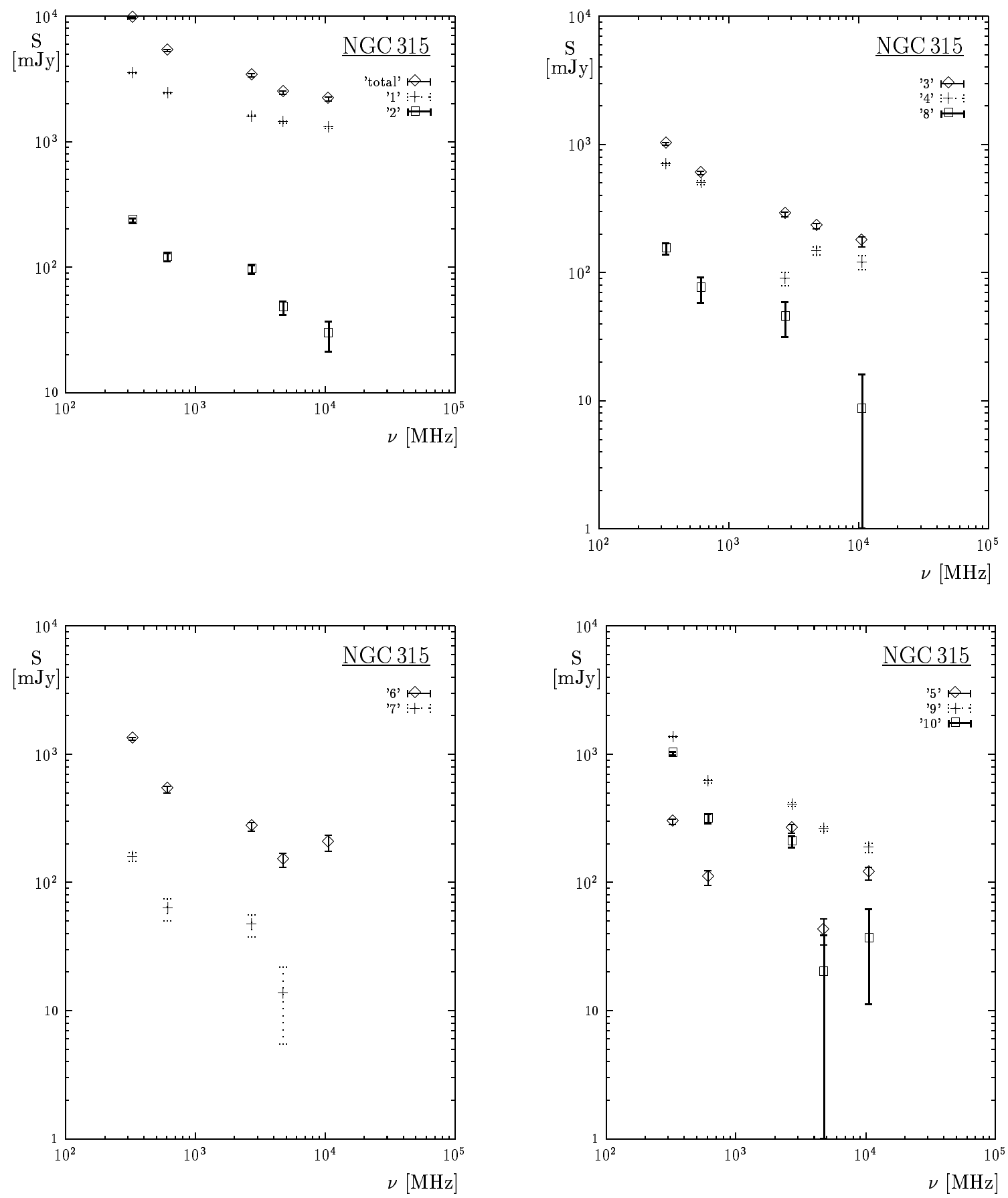

Fig. 52. Spectra of NGC 315 


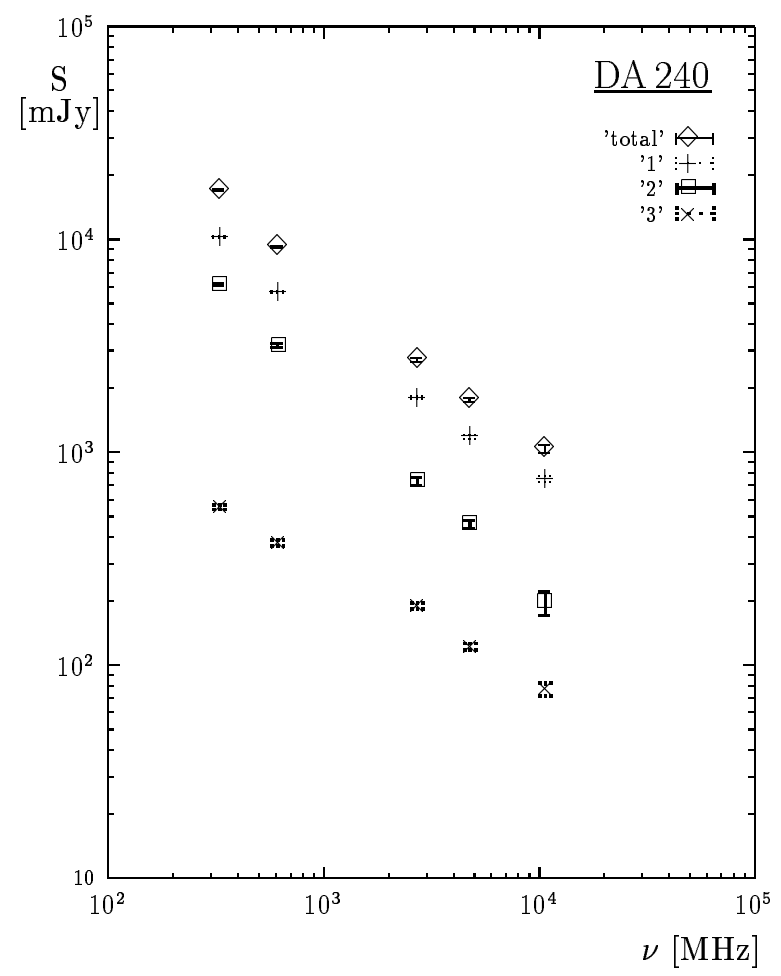

Fig. 53. Spectra of DA 240
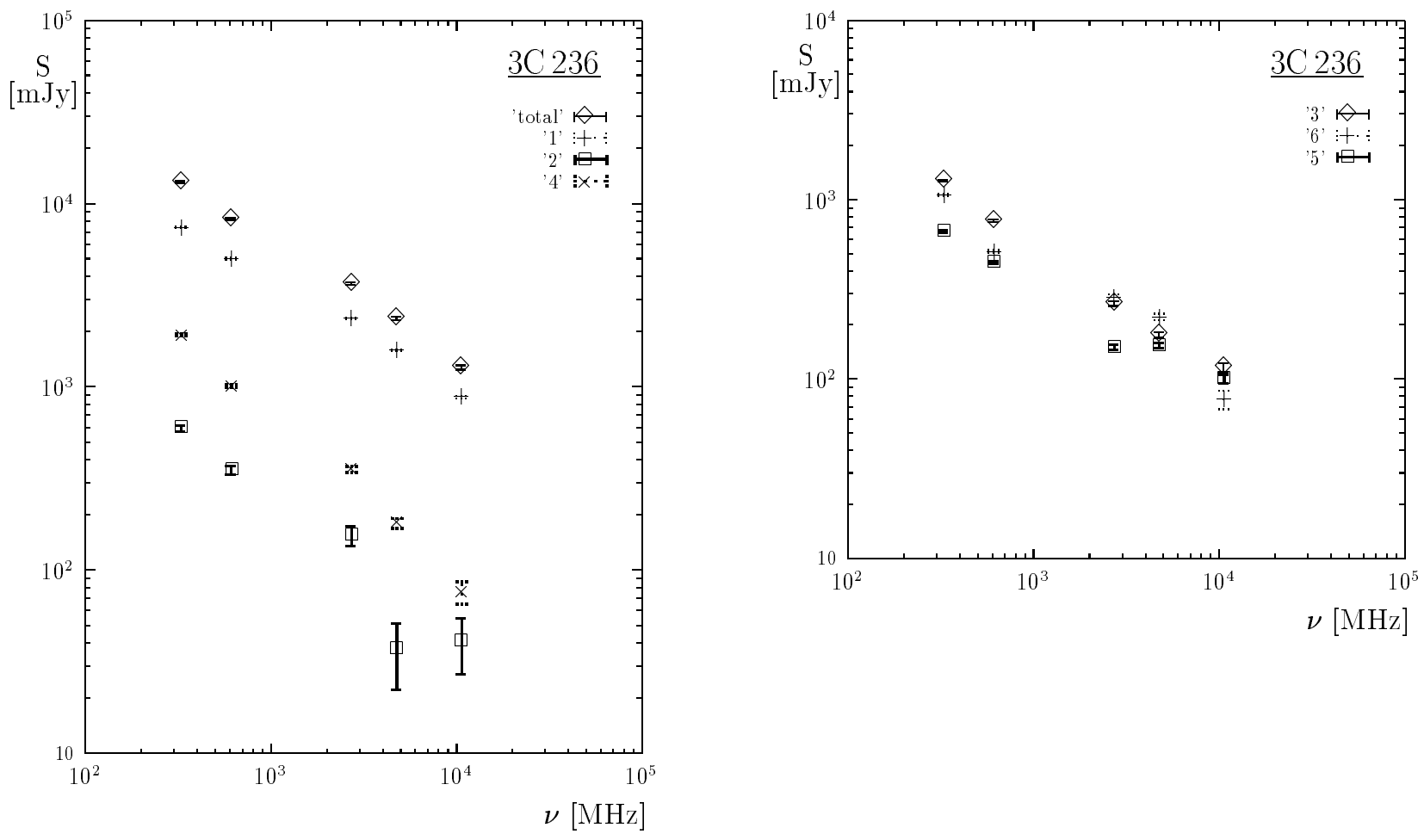

Fig. 54. Spectra of $3 \mathrm{C} 236$ 


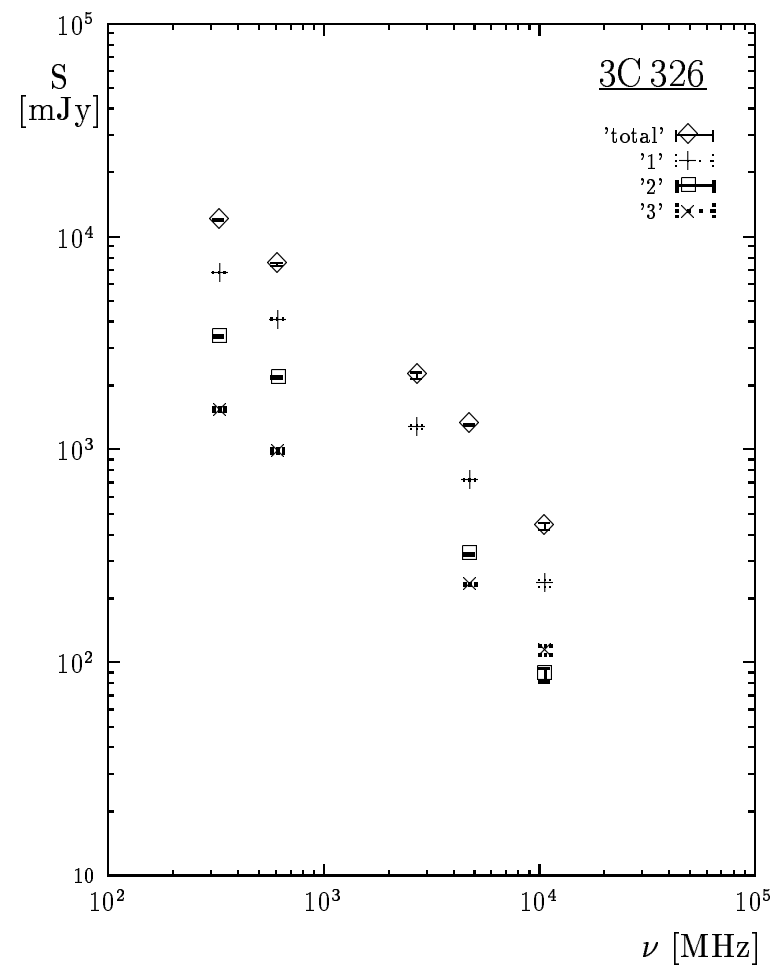

Fig. 55. Spectra of $3 \mathrm{C} 326$
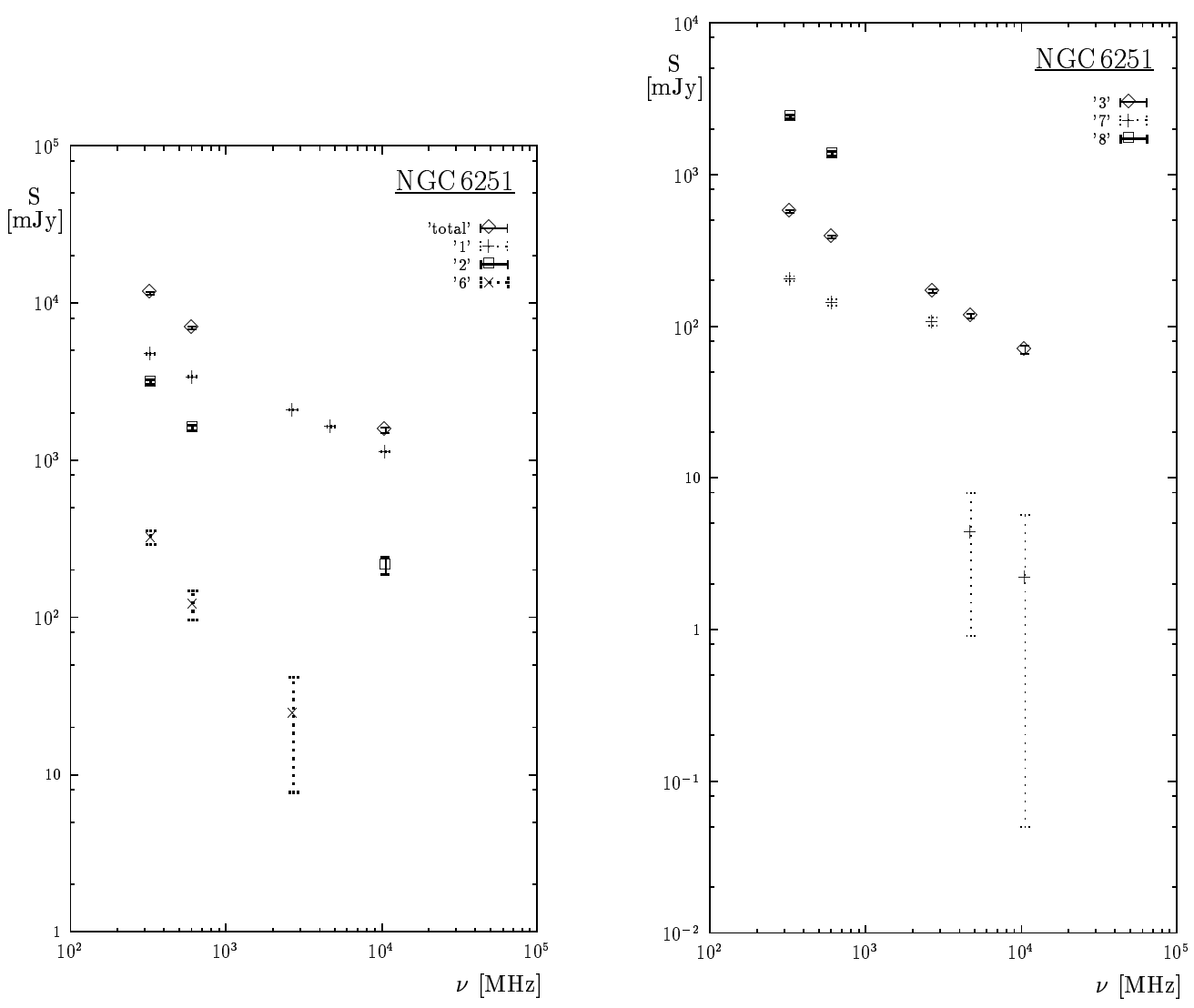

Fig. 56. Spectra of NGC 6251 